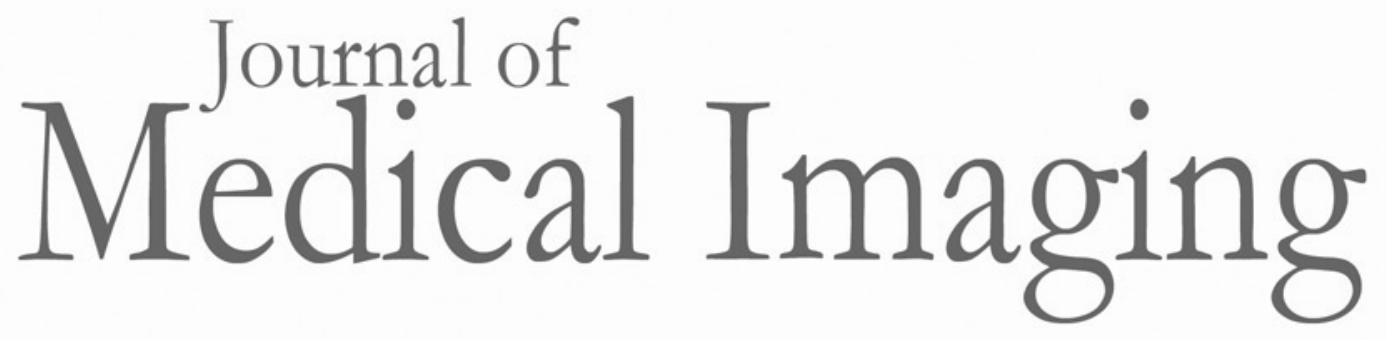

\title{
Reproducibility of an airway tapering measurement in computed tomography with application to bronchiectasis
}

\author{
Kin Quan \\ Ryutaro Tanno \\ Rebecca J. Shipley \\ Jeremy S. Brown \\ Joseph Jacob \\ John R. Hurst \\ David J. Hawkes
}




\title{
Reproducibility of an airway tapering measurement in computed tomography with application to bronchiectasis
}

\author{
Kin Quan, ${ }^{a, \star}$ Ryutaro Tanno, ${ }^{a}$ Rebecca J. Shipley, ${ }^{b}$ Jeremy S. Brown, ${ }^{c}$ Joseph Jacob, ${ }^{\text {a,c }}$ John R. Hurst, ${ }^{c}$ \\ and David J. Hawkes ${ }^{a}$ \\ aUniversity College London, Center for Medical Image Computing, London, United Kingdom \\ bUniversity College London, Department of Mechanical Engineering, London, United Kingdom \\ 'University College London, UCL Respiratory, London, United Kingdom
}

\begin{abstract}
We propose a pipeline to acquire a scalar tapering measurement from the carina to the most distal point of an individual airway visible on computed tomography (CT). We show the applicability of using tapering measurements on clinically acquired data by quantifying the reproducibility of the tapering measure. We generate a spline from the centerline of an airway to measure the area and arclength at contiguous intervals. The tapering measurement is the gradient of the linear regression between area in log space and arclength. The reproducibility of the measure was assessed by analyzing different radiation doses, voxel sizes, and reconstruction kernel on single timepoint and longitudinal CT scans and by evaluating the effect of airway bifurcations. Using 74 airways from 10 CT scans, we show a statistical difference, $p=3.4 \times 10^{-4}$, in tapering between healthy airways $(n=35)$ and those affected by bronchiectasis $(n=39)$. The difference between the mean of the two populations is $0.011 \mathrm{~mm}^{-1}$, and the difference between the medians of the two populations was $0.006 \mathrm{~mm}^{-1}$. The tapering measurement retained a $95 \%$ confidence interval of $\pm 0.005 \mathrm{~mm}^{-1}$ in a simulated $25 \mathrm{mAs}$ scan and retained a $95 \%$ confidence of $\pm 0.005 \mathrm{~mm}^{-1}$ on simulated CTs up to 1.5 times the original voxel size. We have established an estimate of the precision of the tapering measurement and estimated the effect on precision of the simulated voxel size and CT scan dose. We recommend that the scanner calibration be undertaken with the phantoms as described, on the specific CT scanner, radiation dose, and reconstruction algorithm that are to be used in any quantitative studies. @ The Authors. Published by SPIE under a Creative Commons Attribution 4.0 Unported License. Distribution or reproduction of this work in whole or in part requires full attribution of the original publication, including its DOI. [DOI: 10.1117/1.JMI.6.3.034003] Keywords: tapering; computed tomography (CT) simulations; reproducibility; airways; CT metrology.
\end{abstract}

Paper 19024RR received Jan. 24, 2019; accepted for publication Aug. 23, 2019; published online Sep. 16, 2019.

\section{Introduction and Purpose}

Bronchiectasis is defined as the permanent dilatation of the airways. Patients with bronchiectasis can suffer severe exacerbations requiring hospital admission and have a poorer quality of life. ${ }^{1}$ Clinicians diagnose bronchiectasis on computed tomography (CT) imaging by visually estimating the diameter of the airway/bronchus and its adjacent pulmonary artery and calculating the broncho-arterial (BA) ratio. A BA ratio $>1$ indicates the presence of bronchiectasis. $^{2}$

Various groups have proposed methods to automatically and semiautomatically compute the BA ratio for bronchiectatic airways. ${ }^{3-5}$ However, use of the BA ratio to diagnose bronchiectasis has two major flaws. First of all, the maximum healthy range of the BA ratio can be 1.5 times size of the artery. ${ }^{6}$ Second, blood vessels can change size as a result of factors including altitude, ${ }^{7}$ patient age, ${ }^{8}$ and smoking status. ${ }^{9}$ This conflicts with the assumption that the pulmonary artery is always at a constant size.

An alternative approach to diagnose and monitor bronchiectatic airways is to analyze the taper of the airways, i.e., the rate of change in the cross-sectional area along the airway. ${ }^{2}$ In patients with bronchiectasis, the airway is dilated and so the tapering rate must be reduced. Airway tapering is difficult to assess visually and to measure manually. As described by Hansell, ${ }^{6}$ the observer would have to make multiple cross-

*Address all correspondence to Kin Quan, E-mail: kin.quan.10@ucl.ac.uk sectional area measurements along the airway. As mentioned by Cheplygina et al, ${ }^{10}$ measuring multiple lumen is a manually exhaustive task and prone to mistakes.

\subsection{Related Work}

There have been various strategies to quantify tapering in the airways. The initial proposed tapering measurements by Odry et al. ${ }^{11}$ were restricted to short lengths of the airways. A segmented airway would be split into four equal parts. Each segment had an array of computed lumen diameters. The tapering was measured as the linear regression of the lumen diameters along the branch. The method shared similarity to Venkatraman et al., ${ }^{12}$ but the diameter measurements were taken across the central half of each branch. Various analyses attempted to measure the taper of airways containing multiple branches. In the work of Oguma et al., ${ }^{13}$ the region of interest from the carina to the fifth generation airway was measured; however, this was only performed in patients with COPD. Finally, Weinheimer et al. ${ }^{14}$ used a graphical model of the airways for their proposed tapering measurement. The graphical model was based on a graphical tree originating at the trachea and extending into distal branches, depending on airway bifurcations. A tapering measure was assigned to the edge of the graph depending on the lumen area and generation. They also proposed a regional tapering measurement based on the segments of the lobes of the lungs. 
The described tapering measurements have two key limitations. First of all, there is no detailed quantification of reproducibility when considering differences in specifications of the CT scanner, or reconstruction kernel, making it difficult to compare tapering statistics from different machines or from the same CT scanner employing different scanning parameters. Second, the region of interest for the tapering measurement was restricted to airways that were segmented using the respective airway segmentation software. Bronchiectasis is a heterogeneous disease-it can affect any area in the lung including the peripheral regions. ${ }^{15}$ Thus to encapsulate the disease in the tapering measurement, one would need to consider the region of interest as the entire airway, from the trachea to the most distal point.

In all the proposed tapering measurements, obtaining the cross-sectional area is a necessary input for the algorithms. There have been various analyses attempting to validate the reproducibility and precision of measurements against dose, ${ }^{16-18}$ voxel size ${ }^{19}$ reconstruction kernel, ${ }^{20-22}$ and normal biological variation. ${ }^{23}$ In most of the validation experiments, area measurements were taken from phantom, ${ }^{20,21}$ porcine, ${ }^{16,18}$ or cadaver ${ }^{24}$ models. Fetita et al. ${ }^{25}$ used synthetic models of the lung. None of these experiments were explicitly performed on scans with bronchiectasis. Furthermore, the area measurements were not taken at contiguous intervals along the lumen thus missing possible dilatations from a bronchiectatic airway.

For our work and the method from Oguma et al., ${ }^{13}$ the tapering measurement involves the computation of the arc length of the airway at contiguous intervals. In the literature, investigations of the reproducibility of arc length computation in airways are limited. The work of Palágyi et al. ${ }^{26}$ used simulated rotation of in vivo scans. The assessment of the reproducibility was based on the lengths of a single branch rather than multiple generations of branches, thereby precluding estimations of reproducibility of airway quantitation from the carina to an airway's most distal point.

\subsection{Contributions of the Paper}

To our knowledge, there is no detailed analysis on the reproducibility of a global tapering measurement of airways using CT. Thus the purpose of this paper is as follows. First of all, we will discuss in detail a tapering measurement of the airways on CT imaging. Second, we quantify the reproducibility of the measurement against variations in simulated dose and voxel sizes. In addition, we compare the variability of the tapering measurements across different CT reconstructions kernel. Finally, we analyze the effect of bifurcations on tapering measurements and consider measurement repeatability using longitudinal scans.

\section{Method}

We first describe in detail the steps to acquire the airway tapering measurement. The method was initially proposed by Quan et al. $^{27}$; it is summarized in Fig. 1. The pipeline required two inputs. First, the most distal point of each airway of interest was manually identified by an experienced radiologist (J. J.).

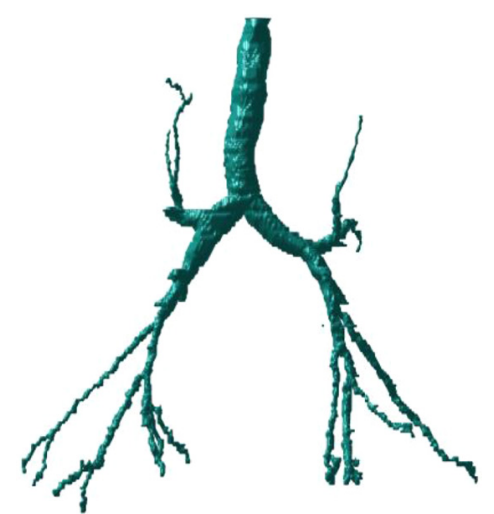

(a)

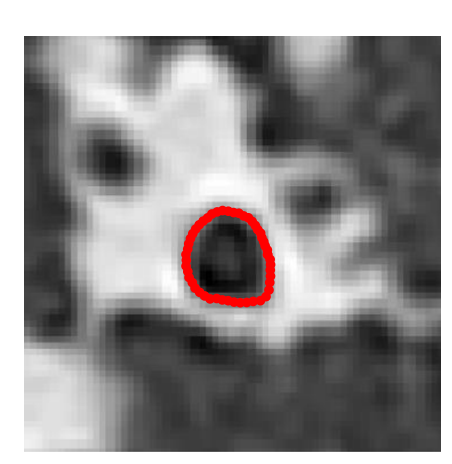

(d)

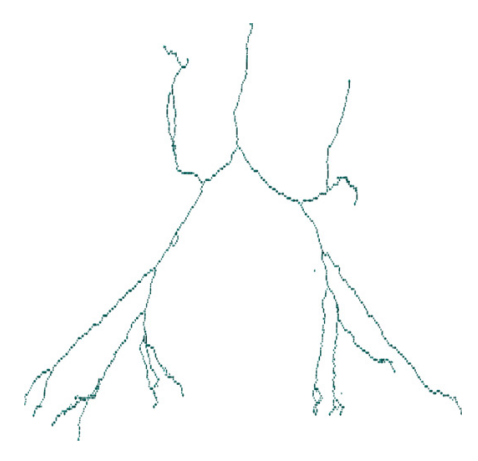

(b)

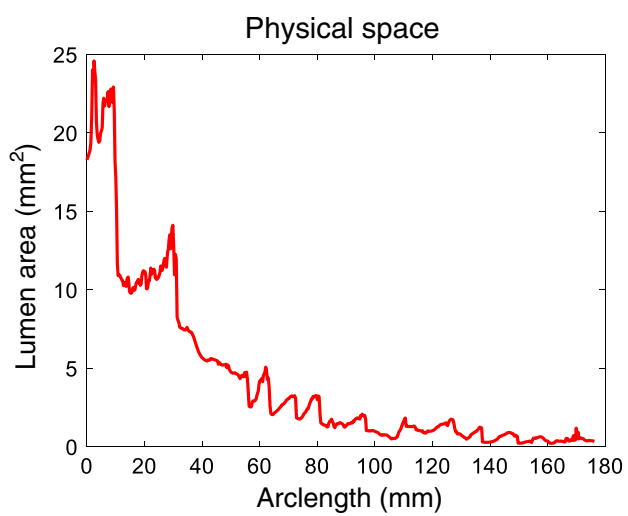

(e)

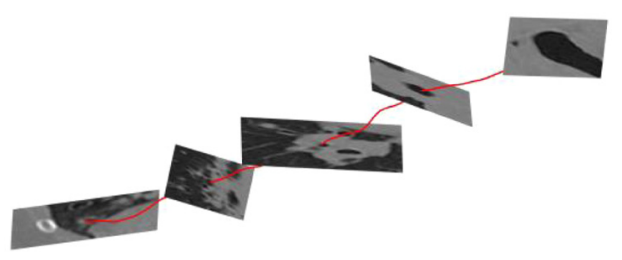

(c)

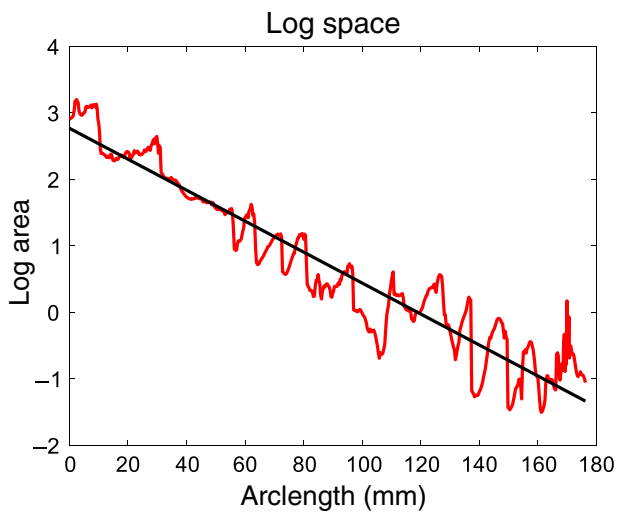

(f)

Fig. 1 Summary of steps in our pipeline: (a) airway segmentation, (b) centerline computation, (c) reconstruct planes on the centerline spline, (d) lumen identification via ray casting, (e) lumen area along the airway, and (f) area profile in log space with line of best fit. 
A single voxel was marked at the end of the airway centerline. The entire analysis was completed using ITK-SNAP. ${ }^{28}$

Second, a complete segmentation of the airway was produced. We obtained an airway segmentation by implementing a method developed by van Rikxoort et al. ${ }^{29}$ The algorithm was based on a region growing paradigm. In summary, a wave front was initialized from the trachea. Voxels on each new iteration were classed as airways based on a voxel criterion. The wave front continued until a wave front criteria was met. In certain cases, the airway segmentation was unable to reach the distal points, and, in these cases, we extended the airway segmentation manually to the distal points. Our method is designed so that it can be incorporated to any system that provides both the segmentation and distal point to the airway of interest. Once the inputs were available, we acquired the measurement using an automatic process.

\subsection{Centerline}

The centerline was used to identify and order the airway segments for the tapering measurement. We implemented a curve thinning algorithm developed by Palágyi et al. ${ }^{26}$ At initialization, the algorithm used the airway segmentation and distal points acquired in Sec. 2. The final input was the start of the centerline at the trachea. The shape of the trachea was assumed to be tubular, with an approximate constant diameter and orientated near perpendicular to the axial slice. Thus the centerline of the trachea lay on the local maximum value of the distance transform of the segmented trachea. ${ }^{30}$ Algorithm 1 was used to find the centerline start point.

\subsection{Recentering and Spline Fitting}

The next task was to separate the centerline of each individual airway from the centerline tree. To this end, we modeled the centerline tree as a graphical model similar to Mori et al. ${ }^{31}$ The nodes corresponded to the centerline voxels and the edges linked neighboring voxels. We performed a breadth first search algorithm $\mathrm{m}^{32}$ on the centerline image. Starting from the carina, we iteratively found the next set of sibling branches. When a distal point was found at the end of a parent branch, the path leading to the distal point was saved. The output was an array of ordered paths describing the unique route from the trachea to the distal point. The proposed tapering measurement started at the carina. Thus centerline points corresponding to the trachea were removed from further analysis.

For each path, we corrected for the discretization errora process known as recentering. ${ }^{33}$ We implemented a similar method to that described by Irving et al. $^{34}$ A five point

Algorithm 1 Locating the start of centerline on the trachea.

Data: $D(i)$ Distance image on the ith axial slice

Result: $\boldsymbol{x}_{\boldsymbol{s}}$ Start point of trachea

$i \leftarrow$ First slice at the top of the trachea.

while $\max D(i)<\max D(i+1)$ do

$i=i+1$

end

$\boldsymbol{x}_{\boldsymbol{s}}=\operatorname{Argmax} D(i)$ smoothing was performed along each path. We modeled the centerline as a continuous model by fitting a cubic spline $F:\left[0, k_{n}\right] \rightarrow \mathbb{R}^{3}$ denoted as

$\boldsymbol{F}(t)=\left\{\begin{array}{ll}\boldsymbol{f}_{1}(t), & t \in\left[0, k_{1}\right] \\ \vdots & \\ \boldsymbol{f}_{n}(t) & t \in\left[k_{n-1}, k_{n}\right]\end{array}\right.$,

where $\boldsymbol{f}_{i}(t)=\sum_{j=0}^{3} c_{i, j} t^{j}$ and $c_{i} \in \mathbb{R}^{3}$. The knots $k_{i}$ where taken on every smoothed point on the centerline. The spline fitting was performed using the $\operatorname{cscvn}{ }^{35}$ function in MATLAB. The continuous model should enable computations of the arc length and tangent at subvoxel intervals along the airway.

\subsection{Arc Length}

The tapering measurement required an array of arc lengths at contiguous intervals from the carina to the distal point. For our pipeline, we considered small parametric intervals $t_{i}$ on the cubic spline $\boldsymbol{F}(t)$. At each interval $t_{i}$, we computed the arc length from the carina to $t_{i}$ as ${ }^{36}$

$s\left(t_{i}\right)=\int_{0}^{t_{i}} \sqrt{\dot{\boldsymbol{F}} \cdot \dot{\boldsymbol{F}}} \mathrm{d} t$,

where $\dot{\boldsymbol{F}}=\frac{\mathrm{d} \boldsymbol{F}}{\mathrm{d} t}$ and $(\cdot)$ is the dot product. For our work, we considered parametric intervals of 0.25 along the spline.

\subsection{Plane Cross Section}

We measured the cross-sectional area accurately by constructing a cross-sectional plane perpendicular to the airway. Using the interval $t_{i}$ from the arc length computation, we computed tangent vector $\boldsymbol{q} \in \mathbb{R}^{3}$ by

$\boldsymbol{q}\left(t_{i}\right)=\frac{\dot{\boldsymbol{F}}}{|\dot{\boldsymbol{F}}|}$.

From linear algebra, points on the plane can be generated by their corresponding basis vector. ${ }^{37}$ To this end, we generated a set of orthonormal vectors $\boldsymbol{v}_{1}, \boldsymbol{v}_{2} \in \mathbb{R}^{2}$ using the method stated by Shirley and Marschner. ${ }^{38}$ The method is summarized in Algorithm 2.

Assuming $\boldsymbol{F}\left(t_{i}\right)$ was the origin, each point $\boldsymbol{u} \in \mathbb{R}^{3}$ on the plane can be written as

$\boldsymbol{u}=\alpha_{1} \boldsymbol{v}_{1}+\alpha_{2} \boldsymbol{v}_{2}$

We selected the scalars $\alpha_{1}, \alpha_{2} \in \mathbb{R}$ such that the point spacing is $0.3 \mathrm{~mm}$ isotropically.

Algorithm 2 Constructing the basis for the plane reconstruction, adapted from Ref. 38.

Data: $\boldsymbol{q}_{1}$ Unit tangent vector of the spline

Result: $\boldsymbol{v}_{1}, \boldsymbol{v}_{2}$ Basis of the orthogonal plane

$\boldsymbol{a} \leftarrow$ Arbitrary vector such that $\boldsymbol{a}$ and $\boldsymbol{q}$ are not collinear

$\boldsymbol{v}_{1}=\frac{\boldsymbol{a} \times \boldsymbol{q}}{|\boldsymbol{a} \times \boldsymbol{q}|}$,

$\boldsymbol{v}_{2}=\boldsymbol{v}_{1} \times \boldsymbol{q}$. 


\subsection{Lumen Cross-sectional Area}

We calculated the cross-sectional area using the edge-cued segmentation-limited full-width half-maximum $\left(\mathrm{FWHM}_{\mathrm{ESL}}\right)$, developed by Kiraly et al. ${ }^{39}$ The method is as follows. The cross-sectional planes were aligned on both the CT image and airway segmentation. The intensities of the plane were computed for both images using cubic interpolation. Fifty rays were cast out in a radial direction, from the center of the plane. Each ray sampled the intensity of the two planes at a fifth of a pixel via linear interpolation. Thus each ray produced two 1-D images with the first from the binary plane $r_{b}$ and second from the CT plane $r_{c}$. We then applied Algorithm 3 to find boundary point $l$.

The final output of the FWHM $\mathrm{ESL}_{\mathrm{EL}}$ was an array of 2-D points corresponding to the edge of the lumen. Finally, we fitted an ellipse based on the least square principle. The method was developed and implemented in MATLAB by Fitzgibbon et al. ${ }^{40}$ We considered the cross-sectional area as the area of the fitted ellipse.

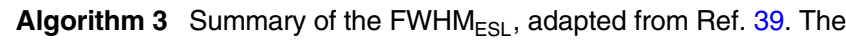
purpose of the algorithm was to find the point of the ray that crossed the lumen.

Data: The rays: $r_{b}:[0, p] \rightarrow \mathbb{R}_{[0,1]}, r_{c}:[0, p] \rightarrow \mathbb{R}$, where $p$ is the length from the center to the border of the plane.

Result: The position of the lumen edge, $I$.

$\mathrm{s} \leftarrow$ The first index of the ray such that $r_{b}(\mathrm{~s})<0.5$

$I_{\text {max }} \leftarrow$ Local maximum intensity in $r_{c}$ nearest to $s$

$x_{\max } \leftarrow$ The index such that $r_{c}\left(x_{\max }\right)=I_{\max }$

$I_{\min } \leftarrow$ Minimum intensity in $r_{c}$ from 0 to $x_{\max }$

$x_{\min } \leftarrow$ The index such that $r_{c}\left(x_{\min }\right)=I_{\text {min }}$

$\underline{I \leftarrow \text { The index such that } r_{c}(I)=\left(I_{\max }+I_{\min }\right) \times 0.5 \text { and } I \in\left[x_{\min }, x_{\max }\right]}$

\subsection{Tapering Measurement}

We assumed for a healthy airway that the cross-sectional area was modeled by an exponential decay along its centerline. It has been shown in human cadaver studies that the average cross-sectional area in a branch reduces at an exponential rate at each generation. ${ }^{41}$ The same observation has been noted in porcine models. ${ }^{42}$ Using the decay assumption, we modeled the relationship between the arc length and the cross-sectional area as

$y=T x+\log A$,

where $x$ is the arc length of the spline, $T$ is the proposed tapering measurement, $y$ is the cross-sectional area, and $A$ is an arbitrary constant.

In terms of implementation, for each airway track, we considered the array arc length and cross-sectional area computed for each individual airway. A logarithmic transform $\log (x)$ was applied only on the cross-sectional area array. We fitted a linear regression on the signal; the tapering measurement is defined as the gradient from the line of best fit.

\section{Evaluation}

An experienced radiologist (J. J.) selected a total of 74 airways from 10 scans. The CT images were analyzed from nine patients with bronchiectasis after obtaining written informed consent at the Royal Free Hospital, London. The voxel size ranged from 0.63 to $0.80 \mathrm{~mm}$ in plane and $0.80-$ to $1.5-\mathrm{mm}$ slice thickness. The airways were classified as healthy $(n=35)$ or bronchiectatic $(n=39)$ by the same radiologist. Details including the make and model of the scanner are provided in Table $1 .^{43}$ From our dataset, many of the airways affected by bronchiectasis came from two patients. We used the same airways for the simulated low-dose and voxel size experiments. A subset of the same airways was used for CT reconstruction kernel and bifurcation experiments.

Table 1 List of CT images used for the experiment. The table includes the number of classified airways, scanner, and voxel size. Abbreviation: GEMD, GE Medical Systems Discovery.

\begin{tabular}{lcccc}
\hline Patients & Bronchiectatic airways & Healthy airways & Scanner & Voxel size $(x, y, z) \mathrm{mm}$ \\
\hline bx500 & 0 & 9 & Toshiba Aquilion ONE & $0.67,0.67,1.50$ \\
bx503 & 15 & 5 & Toshiba Aquilion ONE & $0.64,0.64,1.50$ \\
bx504 & 0 & 8 & Toshiba Aquilion ONE & $0.78,0.78,1.50$ \\
bx505 & 5 & 0 & Toshiba Aquilion ONE & $0.75,0.75,0.80$ \\
bx507 & 0 & Toshiba Aquilion ONE & $0.63,0.63,1.50$ \\
bx508 & 1 & GEMD CT750 HD & $0.80,0.80,1.00$ \\
bx511 & 0 & 0 & Toshiba Aquilion ONE & $0.78,0.78,1.50$ \\
bx512 & 1 & 6 & GEMD CT750 HD & $0.69,0.69,1.00$ \\
bx513 & 1 & 0 & Toshiba Aquilion ONE & $0.73,0.73,1.50$ \\
bx515 & 16 & 3 & Toshiba Aquilion ONE & $0.78,0.78,1.50$ \\
\hline
\end{tabular}




\subsection{Simulated Images}

In this experiment, we simulated images with differing radiation dose and voxel size. The purpose was to analyze the reproducibility of the tapering measurement against various properties of the CT image. Furthermore, we varied the noise and voxel sizes at regular intervals. Thus we also analyzed the sensitivity of the tapering measurement against the given parameters. Finally, we investigated the reproducibility of cross-sectional area and airway length measurements with changes in dose and voxel sizes, respectively.

\subsubsection{Dose}

To simulate the images acquired with different radiation doses, we used the method adapted from Ref. 44. We performed a Radon transform on each axial slice of the original CT image. The output is a sinogram of the respective axial slice. To simulate different radiation doses, Gaussian noise was added on each sinogram with standard deviation $\sigma=10^{\lambda}$, with a range of $\lambda$. The noisy sinograms were then transformed back into physical space using the filtered back projection. The final output is a noisy CT image in Hounsfield units in integer precision. A MATLAB implementation is displayed in Algorithm 4. For

Algorithm 4 Adapted MATLAB code to simulate noise from differing doses.

function noisySlice $=$ AddingDoseNoise (axialSlice, lambda)

$\%$ Creating the sinogram

sinogram = radon(axialSlice, $0: 0.1: 179)$;

\%Adding the Gaussian Noise

noisySinogram $=$ sinogram $+\operatorname{randn}(\operatorname{size}(\text { sinogram }))^{\star} 10^{\wedge}($ lambda $)$;

\%Converting the noisy sinogram into physical space using Filter Backprojection.

noisySlice $=$ iradon(noisySinogram,0:0.1:179, length(axialSlice));

$\%$ Converting into integer precision intensities

noisySlice = int16(noisySlice);

end
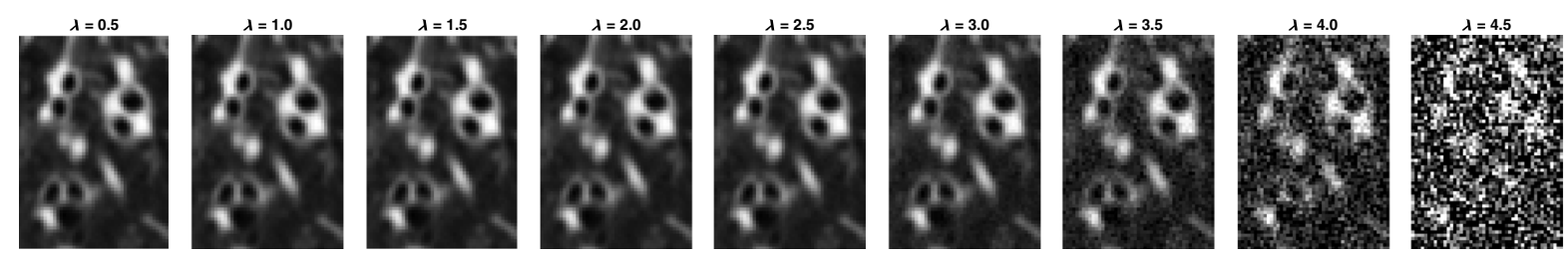

(a)
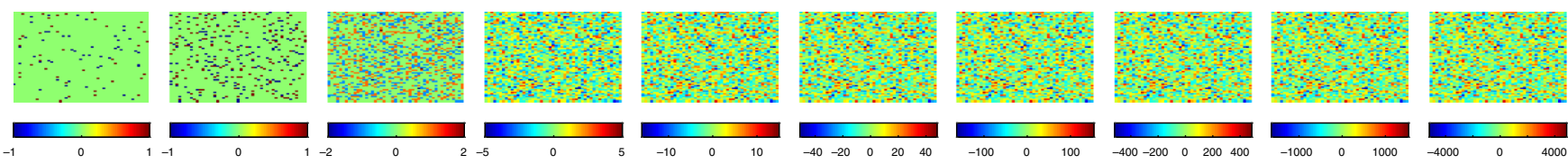

(b)

our experiment, we varied $\lambda$ from 0.5 to 5 in increments of 0.5 . example of the output image is displayed in Fig. 2.

To relate $\lambda$ to the physical dose from a CT scanner, we apted the method described by Reeves et al. ${ }^{45}$ This paper quantified the dose of an image with a homogeneous region region inside the trachea. Using the airway segmentation, we considered the first 60 axial slices of the segmented trachea. To avoid the influence of the boundary, the tracheas were morphologically eroded ${ }^{46}$ with a structuring element of a sphere of processing. Finally, we computed the standard deviation of values of $T_{n}$ on a selection of images against a range of $\lambda$. Using results from Reeves et al. ${ }^{45}$ and Sui et al., ${ }^{47}$ a low-dose scan with a tube current-time product $25 \mathrm{mAs}$ has maximum $T_{n}$ to a low-dose scan. We considered higher values of $\lambda$ to verify y correlations in the results.

using the samed the taper measureme were identified on the respective original image. The literature has shown in low-dose scans, airway segmentation software ${ }^{29,48}$ cannot segment airways to the lung periphery as well as standard dose scans of the same patient. But these methods can still segment a large number of branches in low- and ultralow-dose Furthermore, research has shown that there are minor differences in the performance of radiologists when attempting

\subsubsection{Voxel size}

We analyzed the effect of voxel sizes on the tapering measurement. For each CT image, the voxel spacing $s_{x}, s_{y}, s_{z}$ was subsampled to new spacing of $\sigma s_{x}, \sigma s_{y}, \sigma s_{z}$, where $\sigma$ is a scalar computed using sinc interpolation with a small amount of smoothing. We chose Sinc interpolation to preserve as much information as possible from the original image. To compute tal point to the same coordinate system using nearest neighbor interpolation. Morphological filtering via a closing operation ${ }^{46}$ was used on segmented airways to remove artefacts caused by the resampling. For our experiment, we used the parameters $\sigma=1.1, \ldots, 2$ with increments of 0.1 .$$
\text { (1) }
$$

(20)

Fig. 2 (a) CT images with simulated noise against varying $\lambda$ and (b) an image subtraction of the simulated noisy image with the original. The units are in $\mathrm{HU}$. 
Table 2 Table of standard deviation of intensity $T_{n}(\mathrm{HU})$ in the inner lumen mask for a selected image against differing $\lambda$.

\begin{tabular}{lcccccccccc}
$\lambda$ & $\mathrm{bx500}$ & $\mathrm{bx503}$ & $\mathrm{bx504}$ & $\mathrm{bx505}$ & $\mathrm{bx507}$ & $\mathrm{bx508}$ & $\mathrm{bx511}$ & $\mathrm{bx512}$ & $\mathrm{bx513}$ & $\mathrm{bx515}$ \\
\hline Ground truth & 15 & 28 & 15 & 21 & 16 & 28 & 20 & 33 & 21 & 17 \\
0.5 & 15 & 28 & 15 & 21 & 16 & 28 & 20 & 33 & 21 & 17 \\
1.0 & 15 & 28 & 15 & 21 & 16 & 28 & 20 & 33 & 21 & 17 \\
1.5 & 15 & 28 & 15 & 21 & 16 & 28 & 20 & 33 & 21 & 17 \\
2.0 & 15 & 28 & 15 & 21 & 16 & 28 & 20 & 33 & 21 & 17 \\
2.5 & 16 & 29 & 16 & 22 & 17 & 28 & 20 & 33 & 22 & 17 \\
3.0 & 21 & 32 & 21 & 26 & 22 & 31 & 25 & 36 & 26 & 22 \\
3.5 & 49 & 54 & 49 & 51 & 49 & 54 & 51 & 57 & 51 & 49 \\
4.0 & 146 & 150 & 148 & 148 & 148 & 148 & 149 & 149 & 148 & 145 \\
4.5 & 461 & 467 & 467 & 463 & 466 & 462 & 467 & 459 & 463 & 457 \\
5.0 & 1456 & 1473 & 1477 & 1464 & 1474 & 1458 & 1475 & 1449 & 1463 & 1445 \\
\hline
\end{tabular}
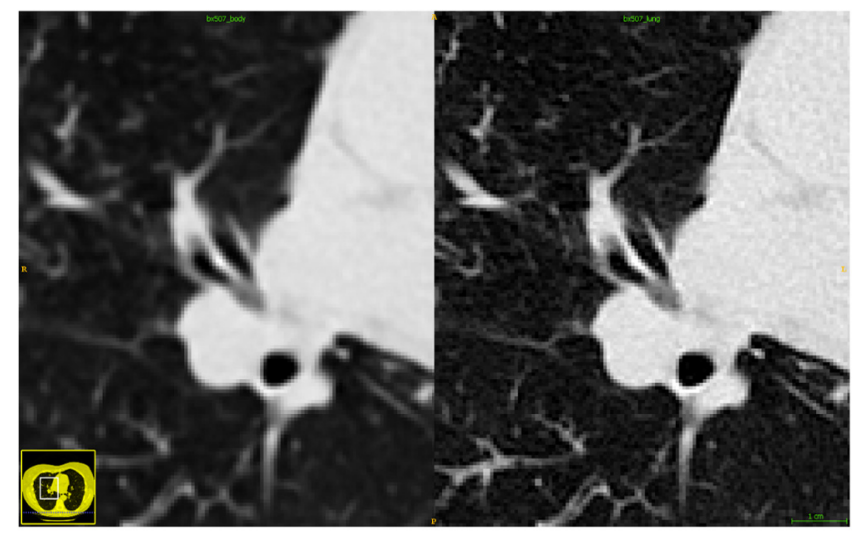

(a)

(b)

Fig. 3 Images from the same CT scan with the (a) body kernel and (b) lung kernel. Both images are displayed in the same intensity window.

\subsection{CT Reconstruction}

On a subset of images, four patients were scanned using the Toshiba Aquilion ONE scanner. On the same scan, two different images were computed. The images were reconstructed using the lung and body kernels, respectively. An example of the reconstruction kernels is displayed in Fig. 3. We acquired the airway segmentation and distal point from a single-reconstruction kernel as described in Table 3. The tapering measurement was computed on both reconstruction kernels using the same airway segmentation and distal points. We used the same airways as described in Table 1.

\subsection{Biological Factors}

\subsubsection{Effect of bifurcations}

We analyzed the effect of airway bifurcations on the tapering measurement. To this end, we manually identified regions of
Table 3 The images used for the reconstruction kernel experiment. This table lists which reconstruction kernel was used to generate the airways segmentation and distal point labeling. The make, model, and voxels size of the images are displayed in Table 1.

\begin{tabular}{ll} 
Patients & Reconstruction kernel used for prepocessing \\
\hline bx503 & Lung \\
bx507 & Body \\
bx513 & Lung \\
bx515 & Body \\
\hline
\end{tabular}

bifurcating airways. On a selected subset of airways, we considered the reconstructed airway image described in Fig. 16. Using ITK-SNAP, the author (K. Q.) started at the cross-sectional plane corresponding to the carina and scrolled toward the distal point. Using visual inspection, the following protocol was developed to identify bifurcations on cross-sectional planes:

1. The scrolling stops when the airway is almost or at the point of separation.

2. The author scrolls back until the airway stops decreasing in diameter. An alternative interpretation is when the airways are about to enlarge due to the bifurcation.

3. Starting at the point of enlargement and scrolling forward, each slice is delineated as a bifurcating region until complete separation of bifurcating airways is reached. The criteria for a complete separation are the lumen wall of both airways that are completely visible and separate. The entire protocol is summarized in Fig. 4.

For our experiment, we selected 19 airways from Table 1. The data consisted of 11 healthy and 8 bronchiectatic airways. The entire analysis was performed on ITK-SNAP.. 


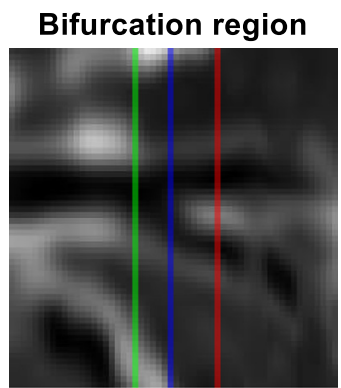

(a)

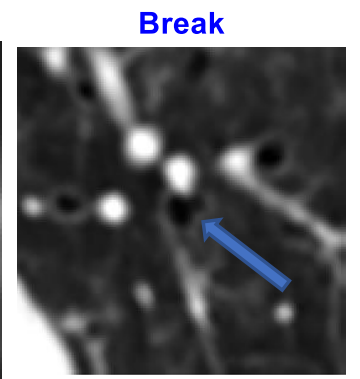

(b)

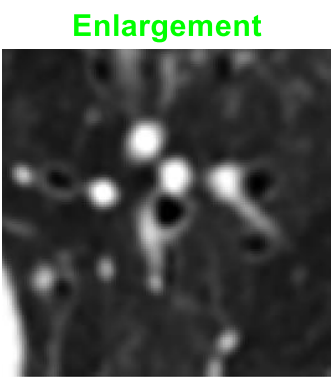

(c)

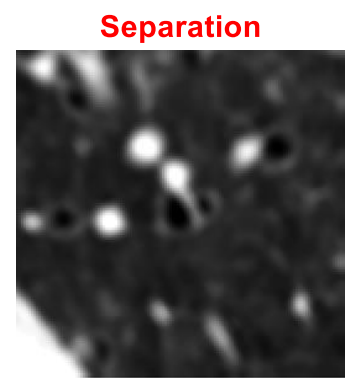

(d)

Fig. 4 (a) A region of bifurcation along the reconstructed slices. The green, blue, and red regions are the slices corresponding to the enlargement, break, and separation slices, respectively. The labeled region consists of slices from green to red. (b) A cross-sectional plane where the airway is at the point of bifurcation, indicated by the blue arrow. (c) First slice of the bifurcation region. (d) The final slice of the bifurcation region. The slides are chronologically ordered with the protocol described in Sec. 3.3.1.

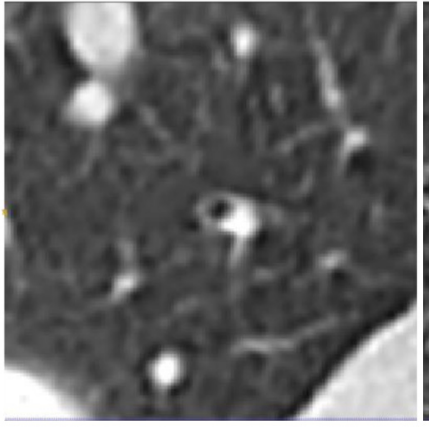

(a)

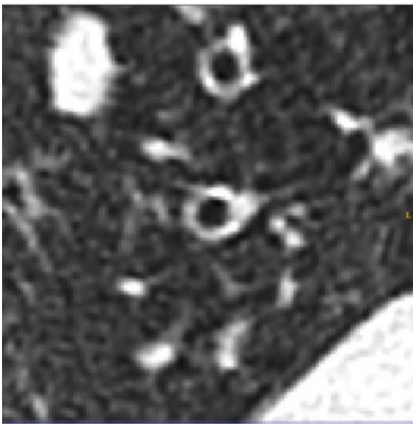

(b)
Fig. 5 The same pair of airways from longitudinal scans: (a) initial healthy airway and (b) the same airway at the same location becoming bronchiectatic.

Table 4 List of the images for progression experiment. This table includes time between scans in months $(M)$ and days $(D)$. The airways on this table are different to Table 1.

\begin{tabular}{lcc}
\hline Patients & Time between scans & Airways labeled \\
\hline bx500 & $9 \mathrm{M} 6 \mathrm{D}$ & 6 \\
bx504 & 35M 6D & 7 \\
bx510 & 5M 22D & 1 \\
\hline P1 & 10M 7D & 5 \\
\hline
\end{tabular}

\subsubsection{Progression}

We examined possible changes in tapering of airways in patients over time. In this experiment, we consider two sets of longitudinal scans. First, pairs of airways that were healthy on both baseline and follow up scans. Second, pairs of airways that were healthy on baseline scans and became bronchiectatic on follow up scans.

For pairs of healthy airways, a trained radiologist (J. J.), manually identified 14 pairs of airways across 3 patients. The criteria were the airway track that must have a healthy appearance on both baseline and follow up scans. For the second population, the same radiologist manually identified 5 pairs of airways from a single patient P1. The scans were obtained from the University College London Hospital and acquired with written consent. The criteria for selection were airways that appear healthy on baseline scans and became bronchiectatic at the follow up scan, an example is displayed in Fig. 5. Details of the CT images are summarized in Tables 4 and 5 .

Using two separate work stations, the airways were visually registered between the longitudinal scans. Airways were taken from various regions of the lungs and were different to the airways displayed in Table 1 . The tapering measurements were taken from the method discussed in Sec. 2.

\section{Results}

Figure 6 compares the tapering measurement between healthy and diseased airways. On a Wilcoxon rank-sum test between the populations, $p=3.4 \times 10^{-4}$.

Table 5 List of make, models, and voxel sizes of CT images for progression experiment. The voxel sizes are displayed as $x, y, z$ and in $\mathrm{mm}$ units. Abbreviations: GEMS, GE medical systems; SS, Siemens SOMATOM.

\begin{tabular}{lcccr}
\hline Patients & Date 1 CT scanner & Date 1 voxel size & Date 2 CT scanner & Date 2 voxel size \\
\hline bx500 & Toshiba Aquilion ONE & $0.67,0.67,1.00$ & Toshiba Aquilion ONE & $0.56,0.56,1.00$ \\
bx504 & Toshiba Aquilion ONE & $0.63,0.63,1.00$ & Toshiba Aquilion ONE & $0.78,0.78,1.00$ \\
bx510 & GEMS Lightspeed Plus & $0.70,0.70,1.00$ & Philips Brilliance 64 & $0.72,0.72,1.00$ \\
\hline P1 & GEMS Discovery STE & $0.85,0.85,2.50$ & SS Definition AS & $0.66,0.66,1.50$ \\
\hline
\end{tabular}




\section{Tapering comparison in} clinical data

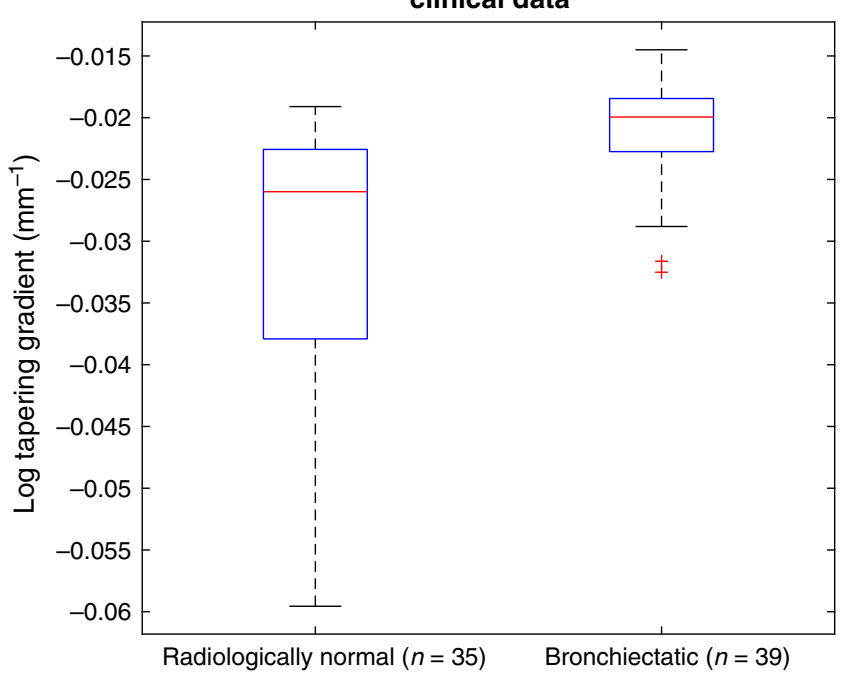

Fig. 6 Comparing the proposed tapering measurement with labeled healthy and bronchiectatic airways. On a Wilcoxon rank-sum test between the populations, $p=3.4 \times 10^{-4}$.

\subsection{Dose}

We analyzed the difference in cross-sectional area measurements and the final tapering measurements at different CT radiation doses.

For the cross-sectional areas, Fig. 7 compares the crosssectional areas between the original image and one of the noisy images. Each graph contains $\sim 30,000$ unique lumen measurements. The correlation coefficients between the populations were $r>0.99$ on all graphs. The $95 \%$ confidence intervals increase with the amount of noise. For the tapering measurement, Fig. 8 displays the measurements from all the noisy images compared to their respective original images. The correlation coefficient between noisy and original tapering measurements was $r>0.98$ on all values of $\lambda$.

We analyzed the tapering difference between the original images and simulated images. We interpret the mean and standard deviation of the error difference as the bias and uncertainty, respectively. Figure 9 shows an overestimation bias with an increase in noise and a positive correlation between uncertainty and dose.

\subsection{Voxel Size}

We analyzed the computed spline and tapering for all the scaled images. We used the arclength of the spline as the metric for comparison for the computed spline. Figure 10 compares the arclengths computed from the scaled splines with the respective originals. On all scales $\sigma$, the correlation coefficients between measurements were $r>0.98$. Furthermore, we analyzed the error difference in arclength. In Fig. 11, the mean difference shows a weak correlation coefficient with $r=0.55$ with scale $\sigma$. The mean difference shows both an overestimation and underestimation bias with the arclength measurement. Figure 11 shows a weak correlation between standard deviation and scale with $r=0.51$.

In terms of the tapering measurement, Fig. 12 compares the tapering values from the scaled images with the respective originals. The correlation coefficients between the scaled and original tapering values was $r>0.97$ on all scales $\sigma$. In addition, we
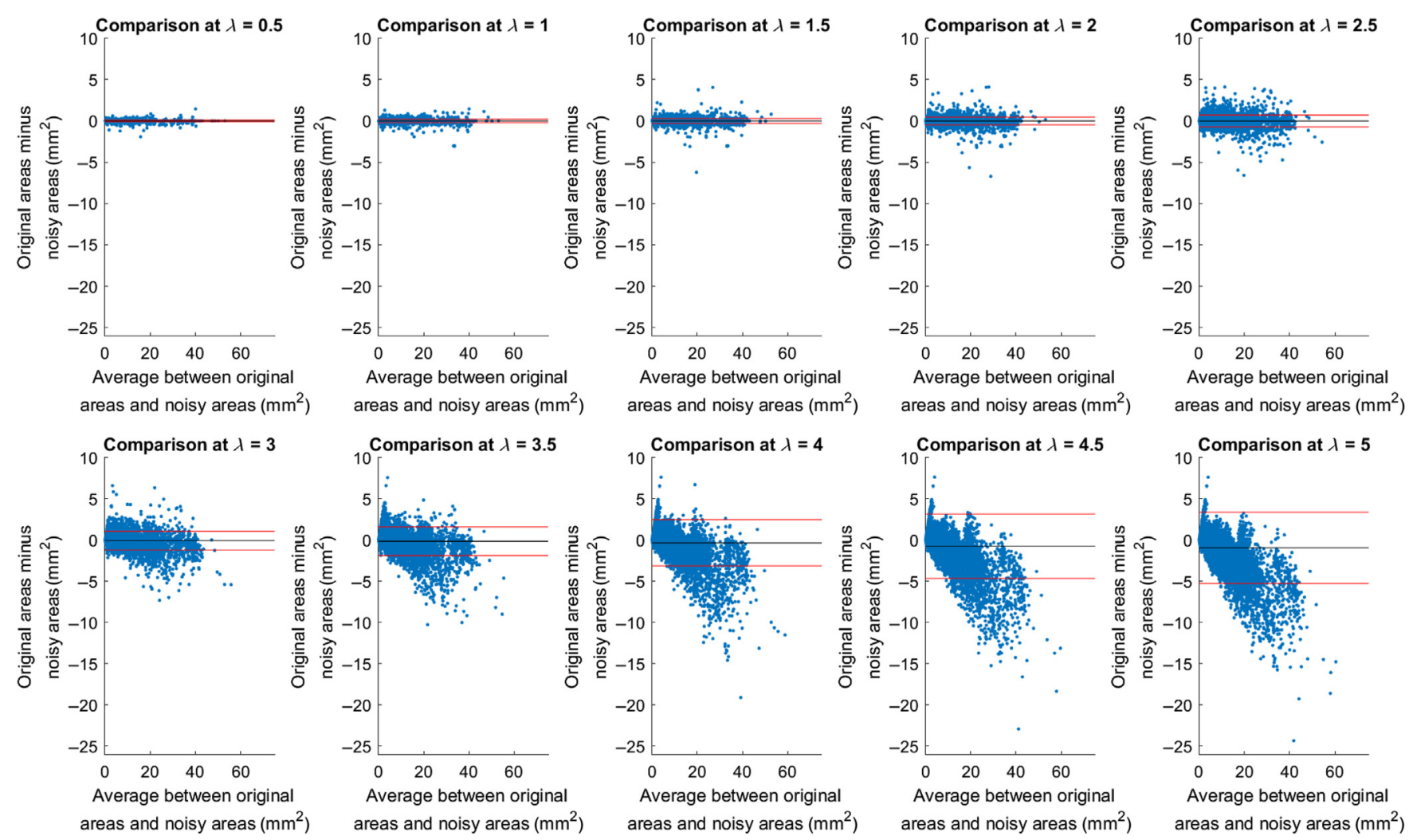

Fig. 7 A series of Bland-Altman ${ }^{54}$ graphs comparing area measurements from a simulated low-dose scan and the original image. On all graphs, the correlation coefficient was $r>0.99$. 

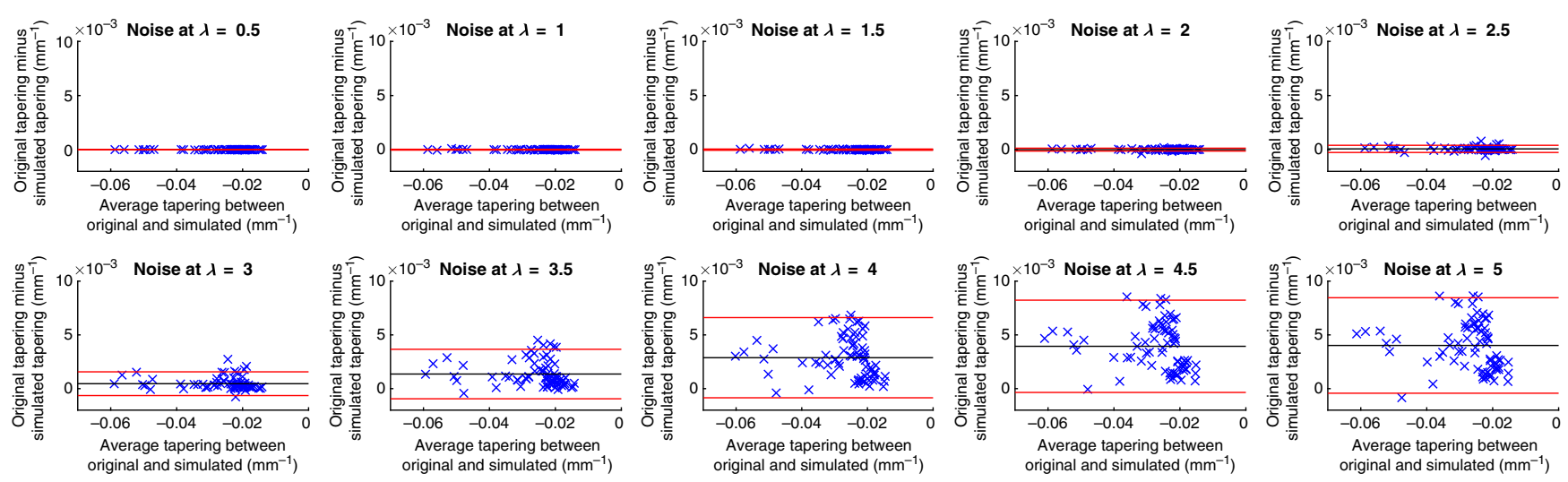

Fig. 8 A series of Bland-Altman ${ }^{54}$ graphs comparing tapering measurement between simulated dose and the original image. On all graphs, the correlation coefficient was $r>0.98$.

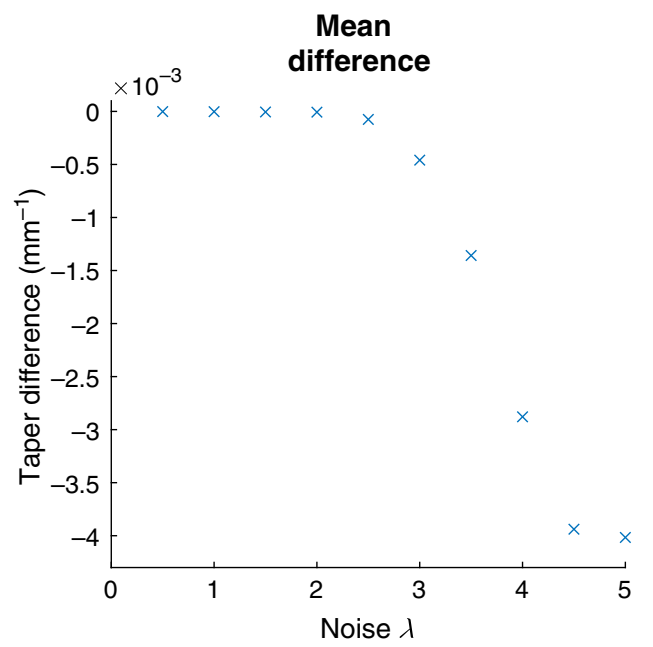

(a)

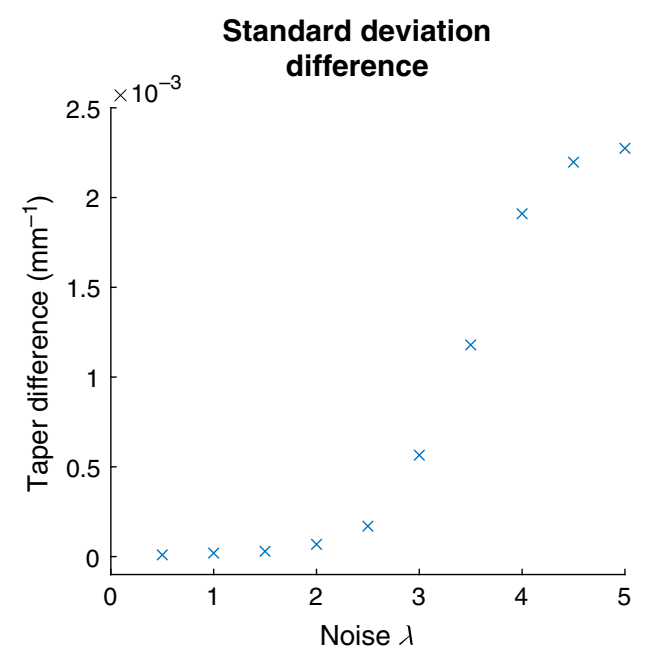

(b)

Fig. 9 (a) Mean and (b) standard deviation of the difference in tapering between original images minus the simulated lower dose.
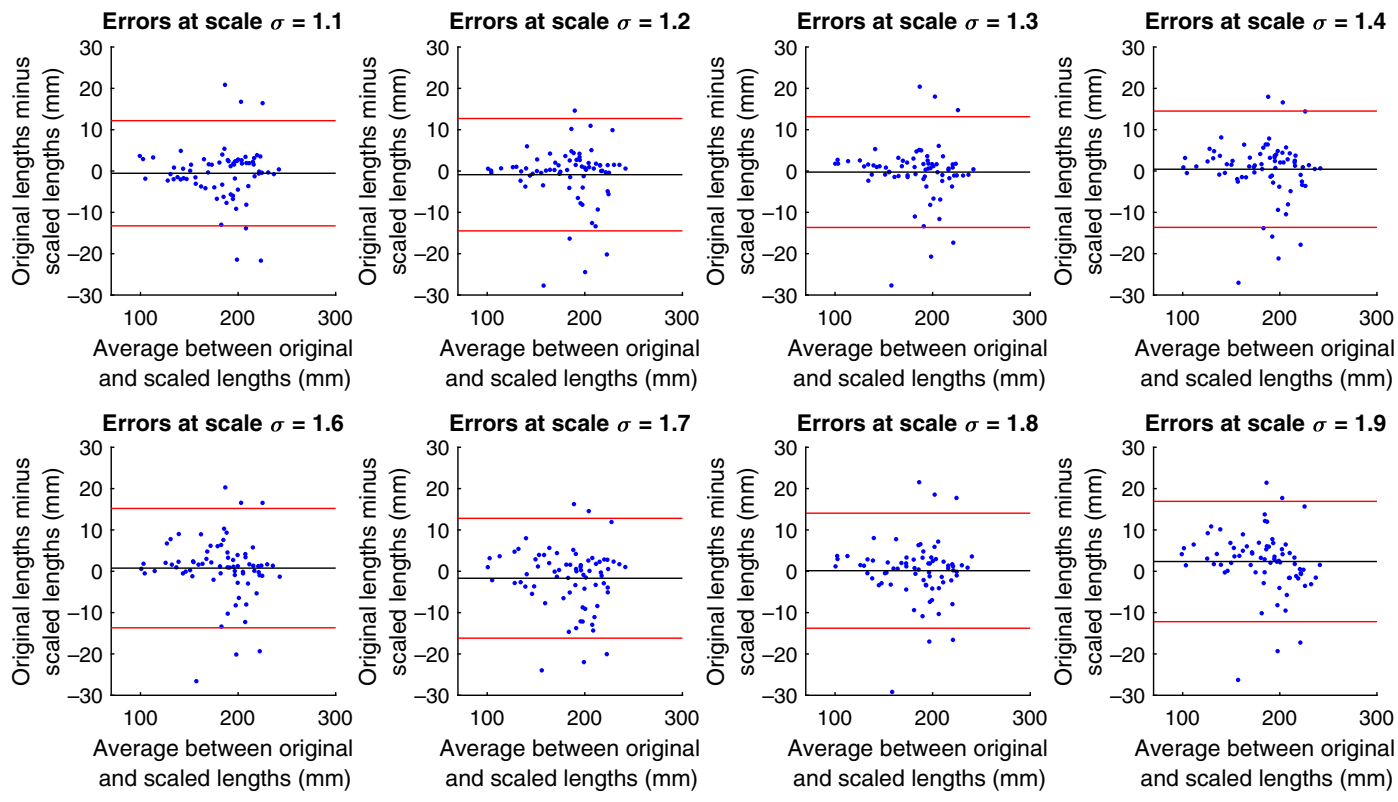

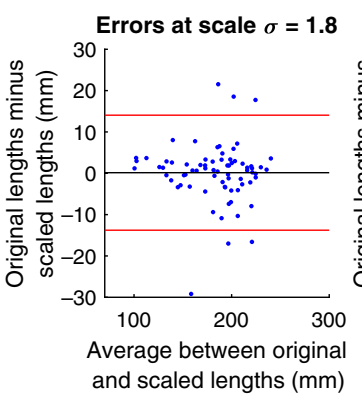

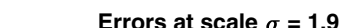

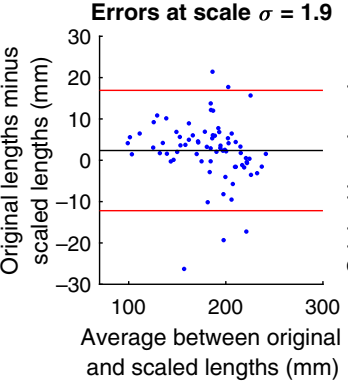

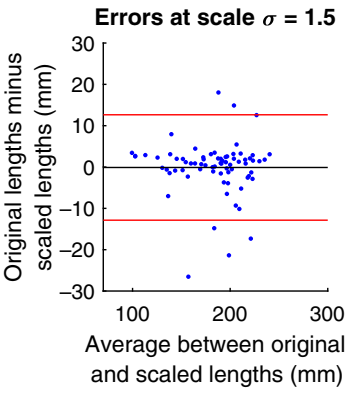

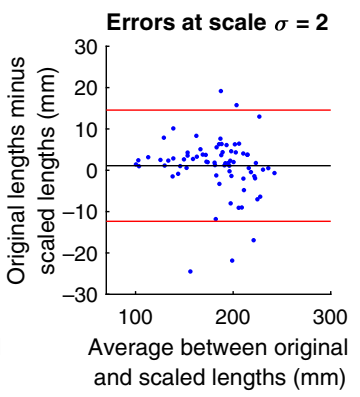

Fig. $10 \mathrm{~A}$ series of Bland-Altman ${ }^{54}$ graphs comparing arc lengths between scaled images and the original images. On all graphs, the correlation coefficient was $r>0.98$. 


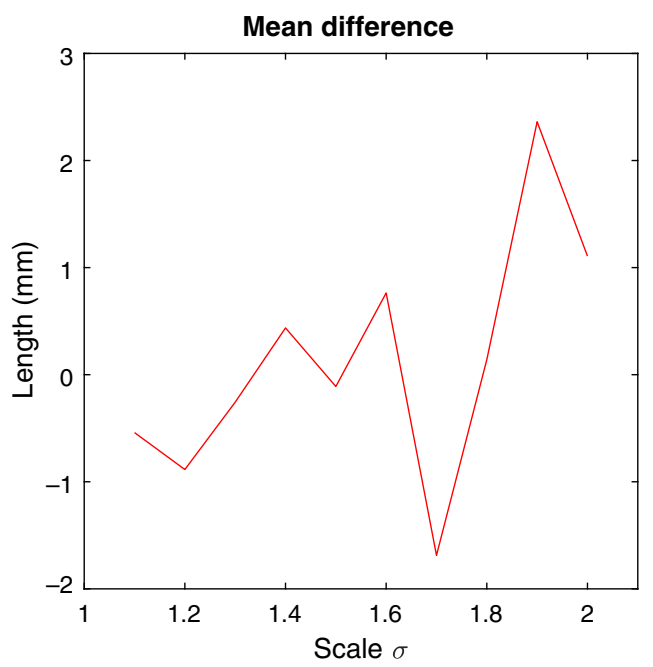

(a)

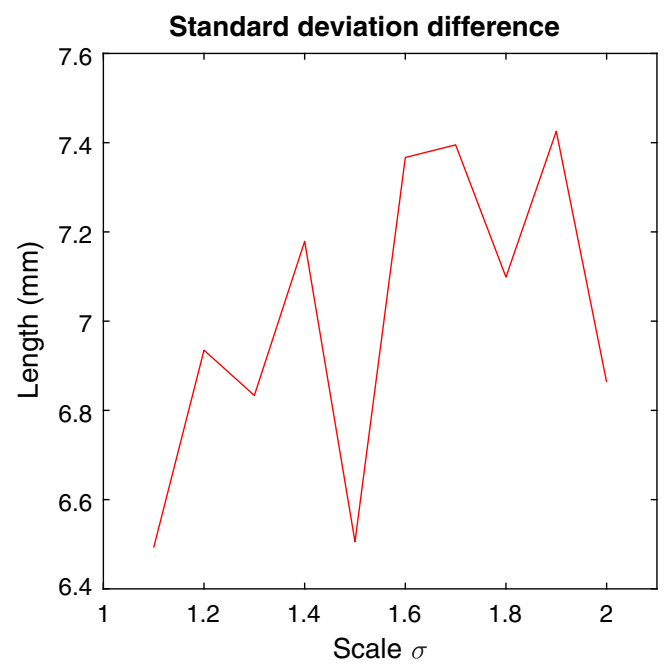

(b)

Fig. 11 (a) Mean and (b) standard deviation of the difference in arclength between original images minus the scaled images. The correlation coefficient of the mean and standard deviation against scale are $r=0.55$ and $r=0.51$, respectively.
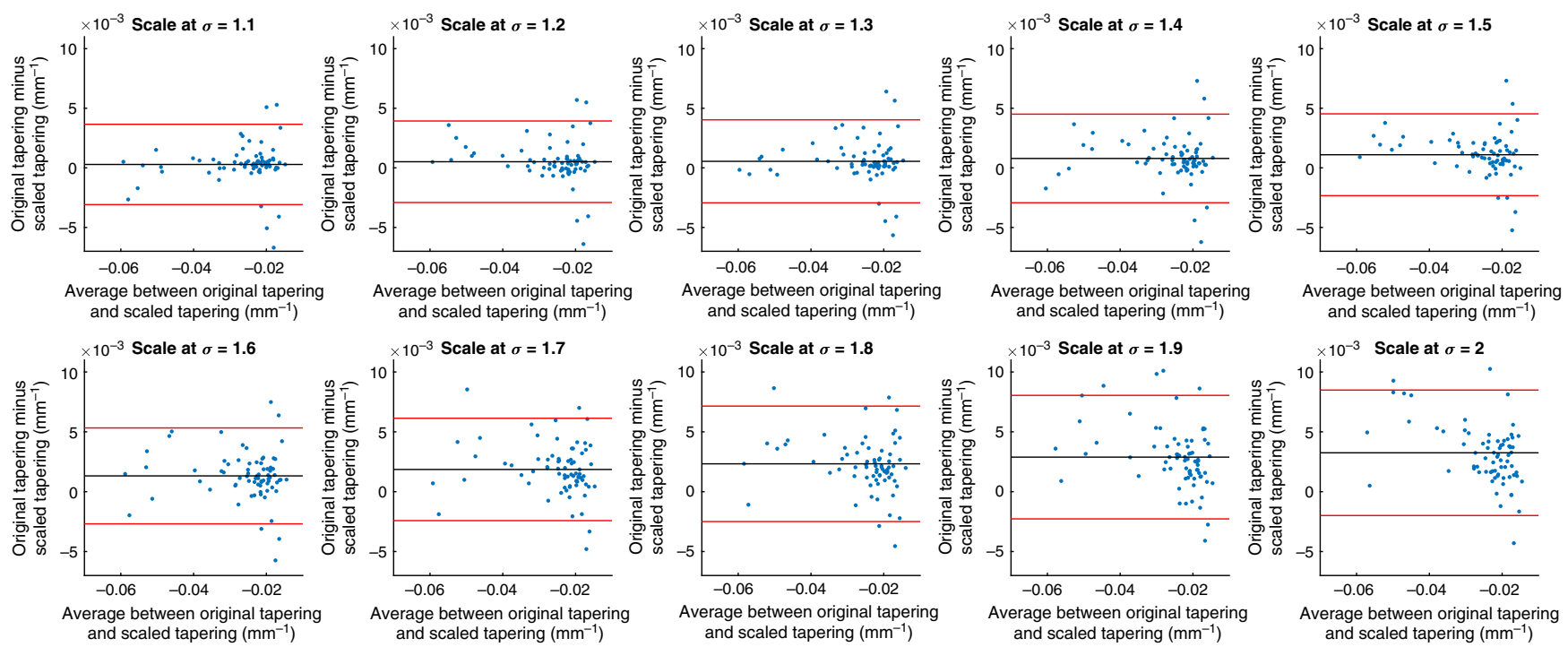

Fig. 12 A series of Bland-Altman ${ }^{54}$ graphs comparing tapering between original images and scaled images. On all graphs, the correlation coefficient was $r>0.97$.

examined the error difference of the original minus the scaled tapering. Figure 13 shows a negative correlation with both overestimation and scale with $r=-0.98$. Furthermore, Fig. 13 shows a positive correlation with uncertainty and scale with $r=0.94$.

\subsection{CT Reconstruction}

We analyzed the difference in cross-sectional area and tapering measurement between reconstruction kernel. Figure 14, compares the difference in area measurements. On all patients, in cross-sectional area measurements, the correlation coefficient between the two measurements was $r>0.99$. The largest $95 \%$ confidence was in patient bx515 with $\pm 1.98 \mathrm{~mm}^{2}$ from the mean. Figure 15 compares the differences in tapering measurement. We collected $n=44$ tapering measurement from
4 patients. The correlation coefficient was $r=0.99$ between the reconstruction kernels.

\subsection{Clinical Results}

\subsubsection{Bifurcations}

We compared tapering measurements with and without points corresponding to bifurcations. On the first dataset, the tapering measurements were computed using all area measurements. The second dataset had tapering measurements computed without area measurements from the bifurcating regions as described in Fig. 16. As we compared the measurements in Fig. 17, the correlation coefficient was $r=0.99$.

The uncertainty of each tapering measurement was computed using the standard error of estimate $s$ defined as ${ }^{55}$ 


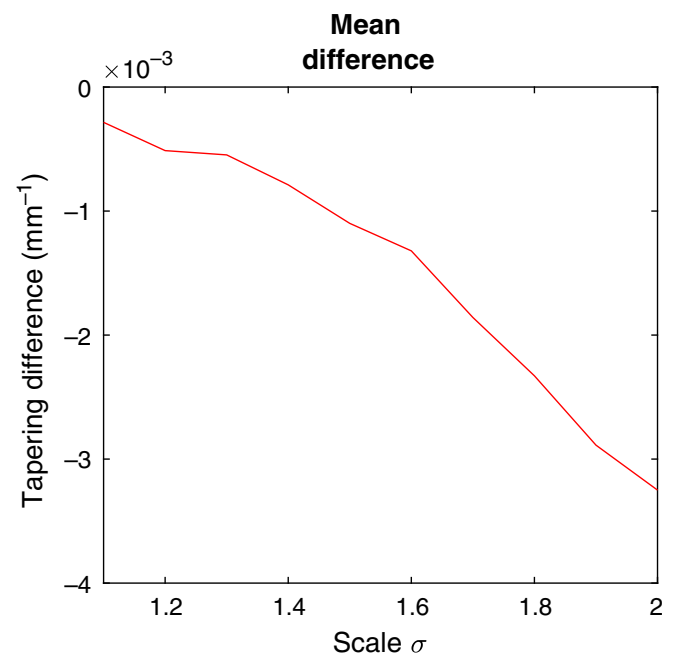

(a)

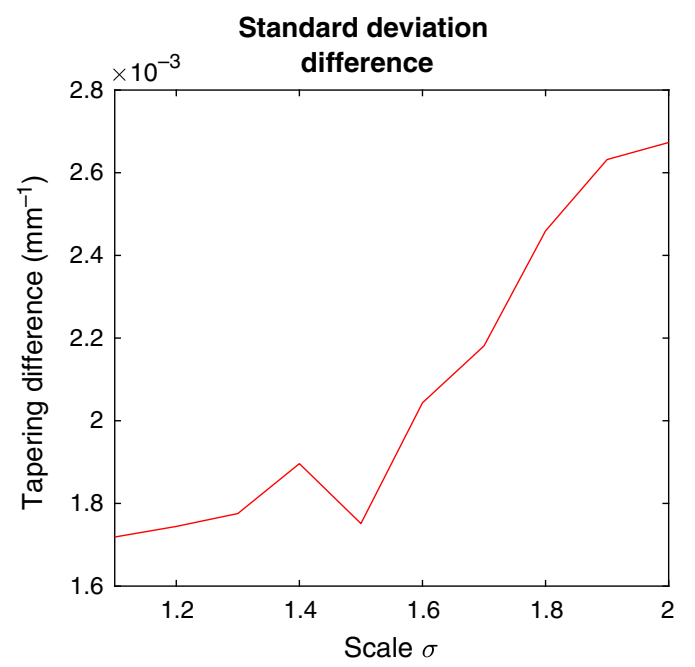

(b)

Fig. 13 (a) Mean and (b) standard deviation of the difference in tapering between original images minus the scaled images. The correlation coefficients of the mean and standard deviation against scale are $r=-0.98$ and $r=0.94$, respectively.
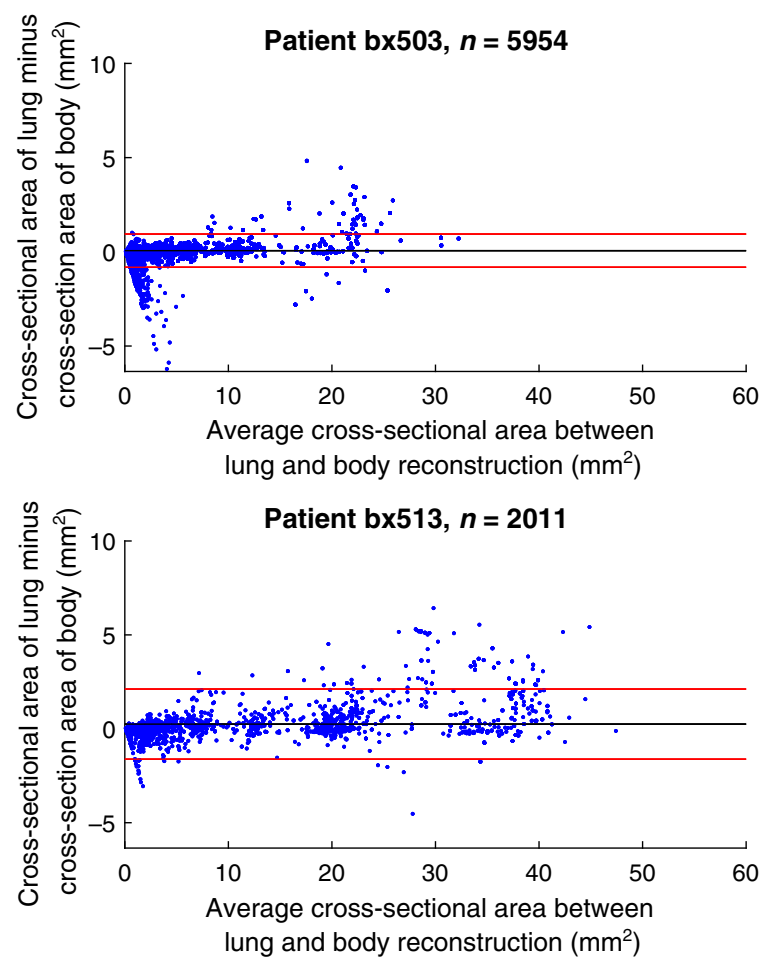
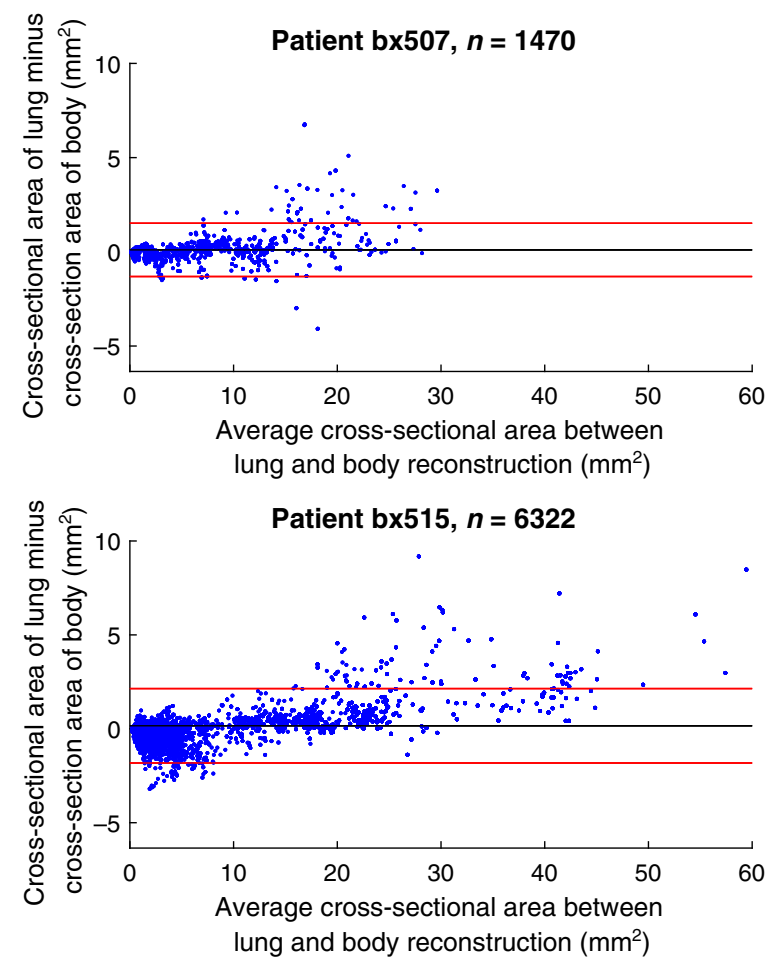

Fig. 14 Bland-Altman ${ }^{54}$ graphs comparing the cross-sectional area between the lung and body kernels. On all four images, the correlation coefficient was $r>0.99$.

$s=\sqrt{\frac{\sum_{i=1}^{N}\left(Y_{i}-y_{i}\right)^{2}}{N}}$,

where $y_{i}$ is the arclength, $Y_{i}$ is the estimate from the linear regression from each computed area $x_{i}$, and $N$ is the number of points in the profile. Figure 16 compares the uncertainty between the two populations. There was a statistical difference between the populations, on a Wilcoxon rank-sum test, $p=$ $7.1 \times 10^{-7}$.

\subsubsection{Progression}

For healthy airways, we grouped tapering values between the baseline and follow up point. Figure 18 compares the measurements between the two time points. The results demonstrated good agreement with an intraclass correlation coefficient ${ }^{56}$ ICC $>0.99$. The standard deviation of the tapering difference was $1.45 \times 10^{-3} \mathrm{~mm}^{-1}$.

For airways that became bronchiectatic, we considered the change in tapering, i.e., tapering value at follow up minus 


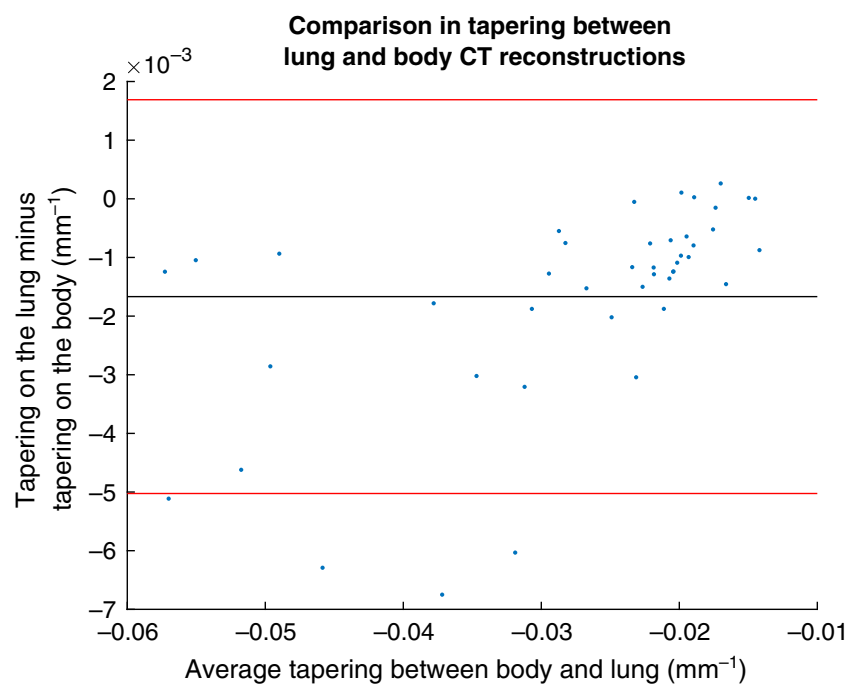

Fig. 15 Bland-Altman ${ }^{54}$ graph comparing tapering measurements $(n=44)$ between lung and body kernels $r=0.99$.

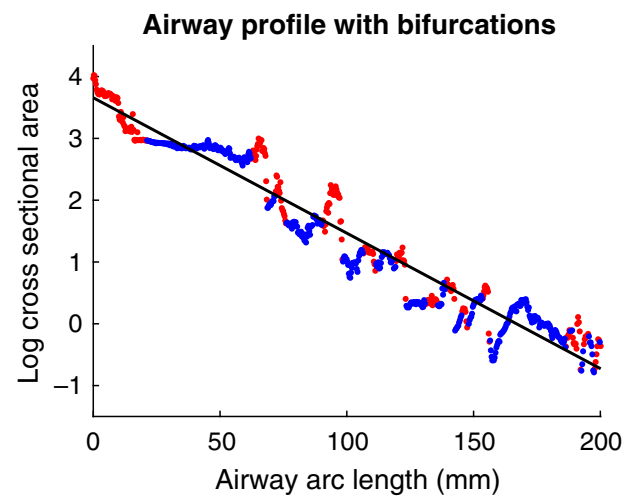

(a)

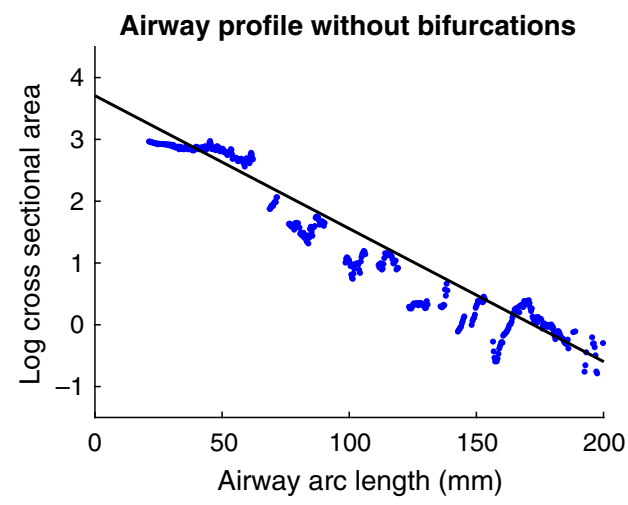

(b)

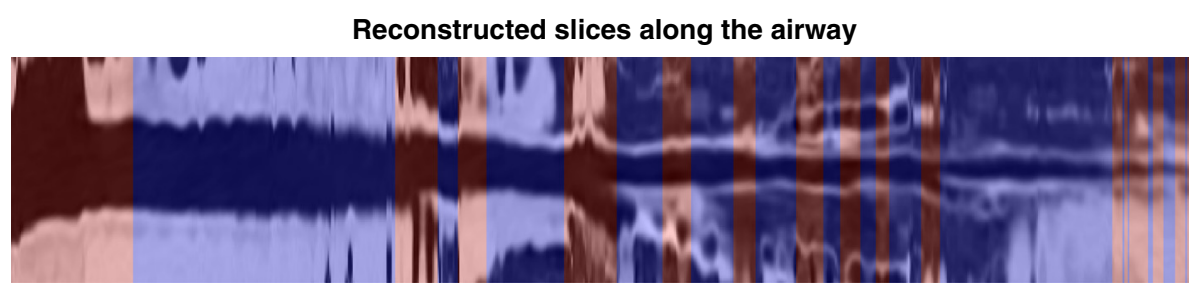

(c)

Fig. 16 (a) A signal of area measurement with bifurcation regions (red) and tubular regions (blue). (b) The same signal with tubular regions (blue) only. On both graphs, the black line is the linear regression of the respective data. The gradient of the line is the proposed tapering measurement. (c) The reconstructed bronchiectatic airway of the same profile. The blue-shaded and red-shaded regions correspond to the tubular and bifurcating airways, respectively. A reconstructed healthy airway has been discussed by Quan et al. ${ }^{27}$ Similar reconstructed cross-sectional images of vessels have been discussed by Oguma et al., ${ }^{13}$ Kumar et al., ${ }^{51}$ Alverez et al., ${ }^{52}$ and Kirby et al. ${ }^{53}$

tapering value at baseline, the results are displayed in Fig. 18. The results show that bronchiectatic airways have a greater tapering change in magnitude compared to airways that remained healthy $p=0.0072$.

\section{Discussions}

In this paper, we propose a tapering measurement for airways imaged using CT and validate the reproducibility of the measurement. The tapering measurement is the exponential decay constant between cross-sectional area and arclength from the carina to the distal point of the airway. Unlike other proposed tapering measurements, we assess reproducibility of the tapering measurement against simulated CT dose, voxel size, and CT reconstruction kernel. Finally, we assess the effect of tapering across airway bifurcations and examine repeatability over time using longitudinal scans.

Part of the evaluations consists of analyzing the difference in tapering across longitudinal scans. The timescales between 


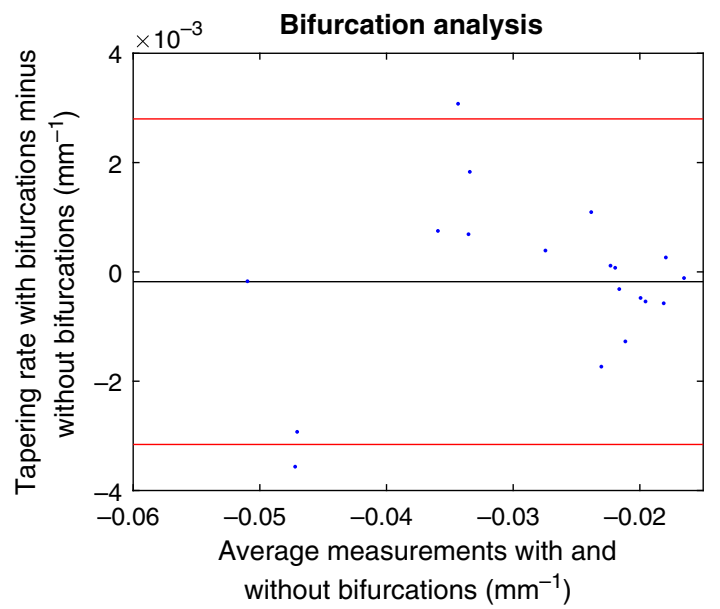

(a)

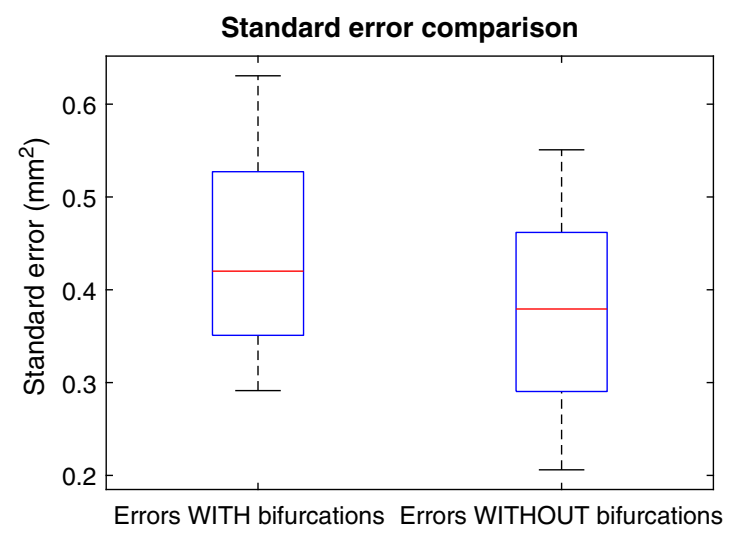

(b)

Fig. 17 (a) Bland-Altman ${ }^{54}$ graph showing the relationship of the taper rates $(n=19)$ with and without bifurcations $r=0.99$ and (b) comparison of the standard error from linear regression between airways with and without bifurcations. On a Wilcoxon rank-sum test between the two populations, $p=7.1 \times 10^{-7}$.

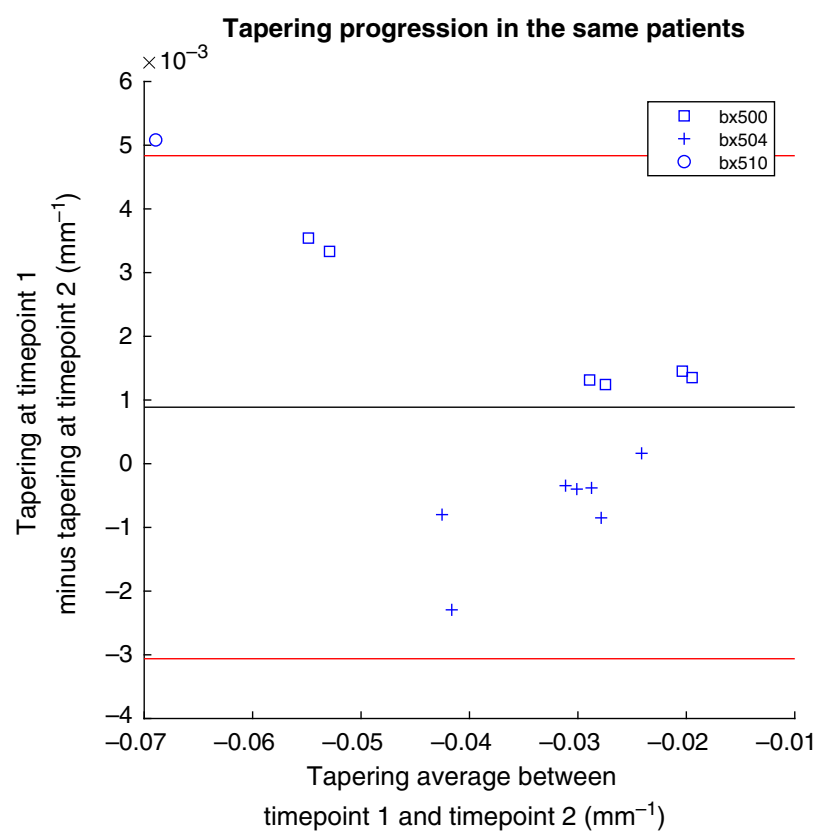

(a)

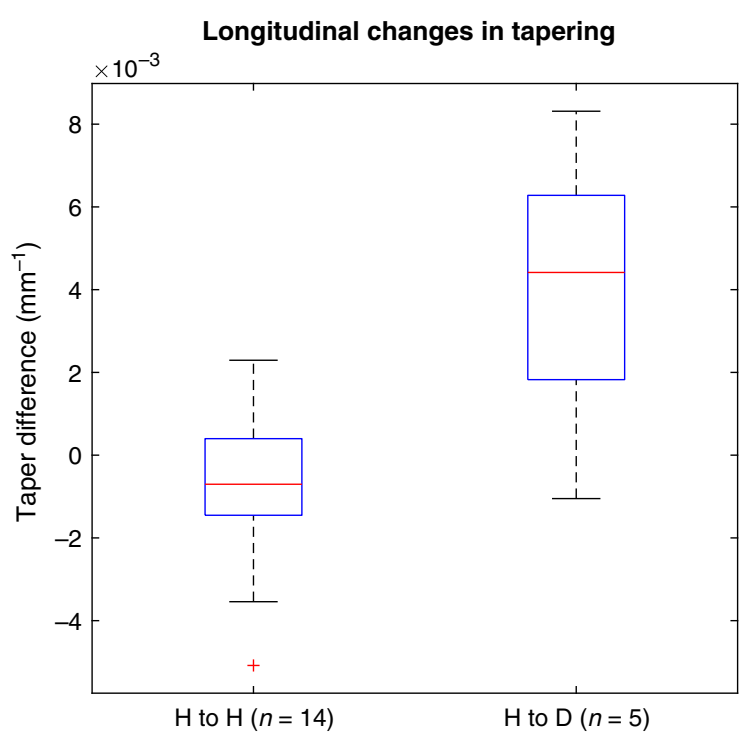

(b)

Fig. 18 (a) Bland-Altman ${ }^{54}$ graph comparing tapering measurement on the same airways from the first and subsequent scan ICC $>0.99$ and (b) The change in tapering between healthy airways $(\mathrm{H}$ to $\mathrm{H})$ and airways that became bronchiectatic on the follow up scans (H to D), $p=0.0072$.

scans range from 9 to 35 months. The motivation for a long timescale is a proof of principle demonstration that the tapering measurement is reproducible for clinical studies. Examples include drug trails ${ }^{57}$ and investigations in exacerbations, ${ }^{58}$ where the timescales in monitoring patients were 12 months and 60 months, respectively.

The pipeline consists of various established image processing algorithms. We chose the centerline algorithm developed by Palágyi et al. ${ }^{26}$ Unlike other proposed methods, ${ }^{30,59,60}$ the algorithm explicitly links the distal points to the carina. Furthermore, it has been shown that the algorithm of Palágyi et al. ${ }^{26}$ can be used on images with nonisotropic voxel sizes. By modeling the centerline as a graphical model similar to Mori et al., ${ }^{31}$ we performed a breadth first search ${ }^{32}$ to avoid analyses of false airway branches. The removal of false branches is not a trivial task. $31,34,61$

We corrected the centerline discretization error or recentering by smoothing points on the centerline. Smoothing has been an established method in the literature. ${ }^{34,62}$ A recentering method was proposed by Kiraly et al., ${ }^{61}$ which shifts the centerline voxels in relation to a distance transform. The process is iterative compared to a single computation of smoothing.

For our pipeline, we generated the orthonormal plane based on the method of Shirley and Marschner. ${ }^{38}$ We set the pixel size 
isotopically at $0.3 \mathrm{~mm}$ to insure that plane image to be within the resolution of the CT image and to allow the ray casting algorithm to find the lumen at subvoxel precision. Other methods have been proposed. In Ref. 36, Kreyszig generated a binormal and principle normal. However, the method is not robust as the binormal vector can become a zero vector. Grélard et al. ${ }^{63}$ used Voronoi cells, a method that requires two parameters whereas Shirley and Marschner ${ }^{38}$ is parameter free. For our work, intensities on the cross-sectional plane were computed via cubic interpolation. Various papers have used linear interpolation. ${ }^{39,64,65}$ However, it has been shown by Moses et al. ${ }^{66}$ that the method can create high-frequency artefacts in the image. ${ }^{67}$

Various methods have been proposed to measure the area of the airway lumen. ${ }^{68-70}$ We used the FWHM ESL $_{\text {because of two }}$ distinct advantages. First, the method is parameter free. Second, the method is robust against slight variations in intensities. The method can, therefore, be applied to images from different scanners and images acquired using different image reconstruction kernels.

\subsection{Limitations}

In this study, we compared the tapering measurement for healthy and diseased airways using a Wilcoxon rank-sum test. The test assumes the data points are independent. However, we used a variety of airways from the same lung. Thus the tapering profiles of the same patients will have a degree of overlap. Future work is needed to analyze data points that are not dependent on each other.

A key limitation of the tapering measurement is the requirement of having a robust airway segmentation. In this paper, the airway segmentation software was often unable to reach the visible distal point of an airway. Thus time-consuming manual delineation was needed to extend the missing airways. The distal point is usually located at the periphery of the lungs. Thus to avoid manual labeling, a segmentation algorithm would need to automatically segment the airways past the sixth airway generation. From the literature, the state-of-the art software developed by Charbonnier et al. ${ }^{71}$ using deep learning could still only consistently segment airways to the fourth generation. The segmentation of small and peripheral airways is not a trivial task. ${ }^{66,72,73}$

In this paper, we analyze the reproducibility of all computerized components of the tapering algorithm. This paper does not address reproducibility of manual labeling of the airways. It is noted in the literature that semimanual labeling of small airways can take hours. ${ }^{74}$ Future work is required to analyze the reproducibility of manual segmentation of the airways. We hypothesize that the segmented healthy peripheral airways consist of a small number of voxels; therefore, any errors in voxel labeling will be considerably smaller then a dilated peripheral airway affected by bronchiectasis.

In this work, we simulated low-dose scans through performing Radon transforms on existing CT images, adding Gaussian noise on the sinogram and using backprojection to reconstruct noisy CT images. There are proposed methods to simulate a low-dose scans by adding a combination of tailored Gaussian and Poisson noise on the sinogram. ${ }^{75}$ These methods assume the original high-dose sinogram are available for simulation; however, it has been acknowledged that sinograms are generally not available in the medical imaging community. ${ }^{76,77}$ Thus various groups have proposed low-dose simulations using reconstructed CT images. The methods involve adding Gaussian $^{76,77}$ or a combination of Gaussian and Poisson noise ${ }^{78}$ on the sinogram of the forward projection of the CT image. Although there has been limited validation of the appearance of lung nodules against simulated low dose simulation, ${ }^{79}$ there has been no validation on the efficacy of these methods on the appearance of airways. We believe that our low-dose simulation is sufficient because the measured standard deviation of the trachea mask $T_{n}$ is similar to results taken from low-dose scans from Reeves et al. ${ }^{45}$ and Sui et al. ${ }^{47}$

Similarly, with voxel size simulation, ideally one would reconstruct the images from the original sinogram. ${ }^{19}$ However, as the sinograms were unavailable, we simulated the voxel size through interpolation of the original CT images similar to Robins et al. ${ }^{80}$ We believe that the simulation is sufficient as it shows the robustness and precision of the centerline, recentering, and crosssectional plane algorithms in the pipeline. Changes in voxel sizes will change the combinatorics or arrangement of the binary image. By showing steps in the pipeline like centerline computation are repeatable across voxel sizes, we avoid resampling the image to isotropic lengths. Thus potentially avoiding a computationally expensive ${ }^{48}$ preprocessing step.

We showed that the tapering measurement is reproducible by measuring the same airway across longitudinal scans with a minimum 5-month interval. The time between scans was on a similar scale from a reproducibility study on airway lumen by Brown et al. ${ }^{23}$ An ideal experiment to assess reproducibility of the same airway from different scans would be to acquire follow up scans immediately after baseline scans similar to Hammond et al. ${ }^{16}$ However, that work was performed on porcine models. Due to considerations of radiation dose, it is difficult to justify the acquisition of additional scans of no clinical benefit. ${ }^{81}$ For our experiment, each airway was chosen by a subspecialist thoracic radiologist. The airway was inspected to ensure it was in a healthy state, e.g., with no mucus present. Thus we assume that each pair of airways is disease free and healthy.

\section{Conclusions}

In this paper, we show a statistical difference in tapering between healthy airways and those affected by bronchiectasis as judged by an experienced radiologist. From Fig. 6, the difference between the mean and median of the two populations was 0.011 and $0.006 \mathrm{~mm}^{-1}$, respectively. In simulated low-dose scans, the tapering measurement retained a $95 \%$ confidence interval of $\pm 0.005 \mathrm{~mm}^{-1}$ up to $\lambda=3.5$, equivalent to a $25-\mathrm{mAs}$ low-dose scan. In simulations assessing different voxel sizes, the tapering measurement retained a $95 \%$ confidence between $\pm 0.005 \mathrm{~mm}^{-1}$ up to $\sigma=1.5$. The tapering measurement retains the same $95 \%$ confidence, $\pm 0.005 \mathrm{~mm}^{-1}$ interval against variations in CT reconstruction kernels, bifurcations, and, importantly, over time in evaluating sequential scans in normal airways. Importantly, we showed as a proof of principle that the magnitude change in tapering for healthy airways is smaller than those from airways that became bronchiectatic. From our previous work, ${ }^{27}$ we showed that the measurements are accurate to a subvoxel level. Our findings suggest that our airway tapering measure can be used to assist in the diagnosis of bronchiectasis, to assess the progression of bronchiectasis with time and, potentially, to assess responses to therapy.

We analyzed the reproducibility of the components that constitute the tapering measurements. The reproducibility of area 
measurements was analyzed in relation to simulated radiation dose and CT reconstruction kernels. For simulated dose, we found the $95 \%$ confidence interval retains $\pm 1.5 \mathrm{~mm}^{2}$ in noisy images under $\lambda=3$, equivalent to a dose just higher than a 25-mAs low-dose scan. We note in Fig. 7, there is a bias toward overestimating larger lumen sizes at lower doses. As the centerline length remains constant and bias on the smaller lumen remain stable, the overestimation results in an increase in taper magnitude. For reconstruction kernels variation, we found the largest $95 \%$ confidence interval was $\pm 1.9 \mathrm{~mm}^{2}$. The reproducibly of arclengths was tested against voxel sizes variability and showed that arclengths have a 95\% confidence interval of up to $\pm 5.0 \mathrm{~mm}$ for scales under $\sigma=1.5$. The increase in the standard deviation of arclength and area against voxel size and dose, respectively, correlate with uncertainty in tapering.

This paper provides useful information for clinical practice and clinical trials. An accurate prediction of the noise amplitude in a particular CT scan and its distribution is a function of the limited radiation dose of the scan, scanner geometry, reconstructed voxel size, other sources of noise, the reconstruction algorithm, and any pre- and postprocessing used. Many of these factors are proprietary information of the CT manufacturer and hence not available to users. ${ }^{82,83}$ We have undertaken an experiment to assess the dependence of our measurements on a simulated noise field added to the CT scan data and have presented the results. This gives an indication of the dependence on radiation dose assuming all other factors remain the same. We recommend that the accuracy experiment presented in this paper be repeated for the particular reconstruction, scan protocol, and scanner type used to make the measurements.

Bronchiectasis is often described as an orphan disease and has suffered a lack of interest and funding. ${ }^{84,85}$ We have shown that the reproducibility of automated airway tapering measurements can assist in the diagnosis and management of bronchiectasis. In addition, we show that it is feasible to use our tapering measurement in large-scale clinical studies of the disease provided careful phantom calibration is taken.

\section{Disclosures}

Part of this work has been presented at the 2018 SPIE Medical Imaging Conference. ${ }^{27}$ For potential conflicts of interest: Ryutaro Tanno has been employed by Microsoft, ThinkSono, and Butterfly Network (the employment is unrelated to the submitted work), Joseph Jacob has received fees from Boehringer Ingelheim and Roche (unrelated to the submitted work), and David Hawkes is a Founder Shareholder in Ixico plc (unrelated to the submitted work). The other authors have no conflicts of interest to declare.

\section{Acknowledgments}

Kin Quan would like to thank Prof Simon Arridge and Dr Andreas Hauptmann for their helpful conversations on the low-dose simulations. This work is supported by the EPSRCfunded UCL Center for Doctoral Training in Medical Imaging (No. EP/L016478/1) and the Department of Health NIHR-funded Biomedical Research Center at the University College London Hospitals. Ryutaro Tanno is supported by Microsoft Research Scholarship. Joseph Jacob is a recipient of Wellcome Trust Clinical Research Career Development Fellowship No. 209553/Z/17/Z.

\section{References}

1. J. D. Chalmers, "Bronchiectasis exacerbations are heart-breaking," Ann. Am. Thorac. Soc. 15(3), 301-303 (2018).

2. M. C. Pasteur, D. Bilton, and A. T. Hill, "British thoracic society guideline for non-CF bronchiectasis," Thorax 65, i1-i58 (2010).

3. C. Fetita et al., "Grading remodeling severity in asthma based on airway wall thickening index and bronchoarterial ratio measured with MSCT," Proc. SPIE 9415, 941515 (2015).

4. E. Ü. Mumcuoglu et al., "Image analysis for cystic fibrosis: computerassisted airway wall and vessel measurements from low-dose, limited scan lung CT images," J. Digital Imaging 26, 82-96 (2013).

5. A. Perez-Rovira et al., "Automatic airway-artery analysis on lung CT to quantify airway wall thickening and bronchiectasis," Med. Phys. 43(10), 5736-5744 (2016).

6. D. M. Hansell, "Thin-section CT of the lungs: the Hinterland of normal," Radiology 256(3), 695-711 (2010).

7. J. S. Kim et al., "Bronchoarterial ratio on thin section CT: comparison between high altitude and sea level," J. Comput. Assisted Tomogr. 21(2), 306-311 (1997).

8. S. Matsuoka et al., "Bronchoarterial ratio and bronchial wall thickness on high-resolution CT in asymptomatic subjects: correlation with age and smoking," Am. J. Roentgenol. 180(2), 513-518 (2003).

9. A. A. Diaz et al., "Quantitative CT measures of bronchiectasis in smokers," Chest 151(6), 1255-1262 (2017).

10. V. Cheplygina et al., "Early experiences with crowdsourcing airway annotations in chest CT," in Int. Workshop Large-Scale Annotation of Biomed. Data and Expert Label Synth. (2016).

11. B. L. Odry et al., "Automated airway evaluation system for multi-slice computed tomography using airway lumen diameter, airway wall thickness and broncho-arterial ratio," Proc. SPIE 6143, 61430Q (2006).

12. R. Venkatraman et al., "Fully automated system for three-dimensional bronchial morphology analysis using volumetric multidetector computed tomography of the chest," J. Digital Imaging 19(2), 132-139 (2006).

13. T. Oguma et al., "Longitudinal shape irregularity of airway lumen assessed by CT in patients with bronchial asthma and COPD," Thorax 70(8), 719-724 (2015).

14. O. Weinheimer et al., "Fully automated lobe-based airway taper index calculation in a low dose MDCT CF study over 4 time-points," Proc. SPIE 10133, 101330U (2017).

15. J. D. Chalmers, S. Aliberti, and F. Blasi, "State of the art review: management of bronchiectasis in adults," Eur. Respir. J. 45(5), 1446-1462 (2015).

16. E. Hammond et al., "Comparison of low- and ultralow-dose computed tomography protocols for quantitative lung and airway assessment," Med. Phys. 44(9), 4747-4757 (2017).

17. Y. Jia et al., "Quantitative analysis of airway tree in low-dose chest CT with a new model-based iterative reconstruction algorithm: comparison to adaptive statistical iterative reconstruction in routine-dose CT," Acad. Radiol. 25(12), 1526-1532 (2018).

18. P. Leutz-Schmidt et al., "Influence of exposure parameters and iterative reconstruction on automatic airway segmentation and analysis on MDCT-An ex vivo phantom study," PLoS One 12(8), e0182268 (2017).

19. T. Achenbach et al., "Influence of pixel size on quantification of airway wall thickness in computed tomography," J. Comput. Assisted Tomogr. 33(5), 725-730 (2009).

20. J. Ken Leader et al., "Quantification of airway morphometry: the effect of CT acquisition and reconstruction parameters," Proc. SPIE 6511 , 65111R (2007).

21. J. C. Wong et al., "The influence of reconstruction algorithm on the measurement of airway dimensions using computed tomography," Proc. SPIE 6916, 691615 (2008).

22. B. Zheng et al., "Automated detection and quantitative assessment of pulmonary airways depicted on CT images," Med. Phys. 34(7), 2844-2852 (2007).

23. R. H. Brown et al., "Reproducibility of airway luminal size in asthma measured by HRCT," J. Appl. Physiol. 123(4), 876-883 (2017).

24. L. Zhang et al., "Airway quantification using adaptive statistical iterative reconstruction-V on wide-detector low-dose CT: a validation study on lung specimen," Jpn. J. Radiol. 37(5), 390-398 (2019). 
25. C. Fetita et al., "Volumetric quantification of airway wall in CT via collision-free active surface model: application to asthma assessment," IEEE Trans. Image Process. 33(7), 1512-1526 (2014).

26. K. Palágyi et al., "Quantitative analysis of pulmonary airway tree structures," Comput. Biol. Med. 36(9), 974-996 (2006).

27. K. Quan et al., "Tapering analysis of airways with bronchiectasis," Proc. SPIE 10574, 105742G (2018).

28. P. A. Yushkevich et al., "User-guided 3D active contour segmentation of anatomical structures: Significantly improved efficiency and reliability," ITK-SNAP Home, 2006, http://www.itksnap.org (accessed 25 July 2019).

29. E. M. van Rikxoort, B. Wouter, and B. Van Ginneken, "Automatic segmentation of the airway tree from thoracic CT scans using a multi-threshold approach," in The First Int. Workshop Pulmonary Image Anal. (2009).

30. S. R. Aylward and E. Bullitt, "Initialization, noise, singularities, and scale in height ridge traversal for tubular object centerline extraction," IEEE Trans. Med. Imaging 21(2), 61-75 (2002).

31. K. Mori et al., "Automated anatomical labeling of the bronchial branch and its application to the virtual bronchoscopy system," IEEE Trans. Med. Imaging 19(2), 103-114 (2000).

32. T. H. Cormen et al., Introduction to Algorithms, MIT Press, Cambridge (2009).

33. F. Grélard et al., "New methods for the geometrical analysis of tubular organs," Med. Image Anal. 42, 89-101 (2017).

34. B. J. Irving et al., "Computer assisted detection of abnormal airway variation in CT scans related to paediatric tuberculosis," Med. Image Anal. 18(7), 963-976 (2014).

35. "Natural" or periodic interpolating cubic spline curve-MATLAB, https:// uk.mathworks.com/help/curvefit/cscvn.html (accessed 25 July 2019).

36. E. Kreyszig, Advanced Engineering Mathematics, John Wiley \& Sons, New York (1964).

37. S. J. Leon, Linear Algebra with Applications, Pearson Prentice Hall, Upper Saddle River, New Jersey (2009).

38. P. Shirley and S. Marschner, Fundamentals of Computer Graphics, A K Peters, Natick, Massachusetts (2009).

39. A. P. Kiraly et al., "Virtual bronchoscopy for quantitative airway analysis," Proc. SPIE 5746, 369-383 (2005).

40. A. W. Fitzgibbon, M. Pilu, and R. B. Fisher, "Direct least squares fitting of ellipses," in Proc. 13th Int. Conf. Pattern Recognit. (1996).

41. A. I. Nikiforov and R. B. Schlesinger, "Morphometric variability of the human upper bronchial tree," Respir. Physiol. 59(3), 289-299 (1985).

42. M. K. Azad, H. A. Mansy, and P. T. Gamage, "Geometric features of pig airways using computed tomography," Physiol. Rep. 4(20), e12995 (2016).

43. B. Milliron et al., "Bronchiectasis: mechanisms and imaging clues of associated common and uncommon diseases," RadioGraphics 35(4), 1011-1030 (2015).

44. D. P. Frush et al., "Computer-simulated radiation dose reduction for abdominal multidetector CT of pediatric patients," Am. J. Roentgenol. 179(2), 1107-1113 (2002).

45. A. P. Reeves, Y. Xie, and S. Liu, "Automated image quality assessment for chest CT scans," Med. Phys. 45(2), 561-578 (2017).

46. R. C. Gonzalez, R. E. Woods, and S. L. Eddins, Digital Image Processing Using MATLAB, Pearson Prentice Hall, Upper Saddle River, New Jersey (2011).

47. X. Sui et al., "Detection and size measurements of pulmonary nodules in ultra-low-dose CT with iterative reconstruction compared to low dose CT,' Eur. J. Radiol. 85(3), 564-570 (2016).

48. R. Wiemker et al., "Unsupervised extraction and quantification of the bronchial tree on ultra-low-dose vs. standard dose CT," Proc. SPIE 6143, 61432V (2006).

49. A. Larbi et al., "Detection and characterization of focal liver lesions with ultra-low dose computed tomography in neoplastic patients," Diagn. Interventional Imaging 99(5), 311-320 (2018).

50. Y. Nagatani et al., "Lung nodule detection performance in five observers on computed tomography (CT) with adaptive iterative dose reduction using three-dimensional processing (AIDR 3D) in a Japanese multicenter study: comparison between ultra-low-dose CT and low-dose CT by receiver-operating characteristic analysis," Eur. J. Radiol. 84(7), 1401-1412 (2015).

51. R. P. Kumar et al., "Three-dimensional blood vessel segmentation and centerline extraction based on two-dimensional cross-section analysis," Ann. Biomed. Eng. 43(5), 1223-1234 (2015).
52. L. Alvarez et al., "Tracking the aortic lumen geometry by optimizing the 3D orientation of its cross-sections," Lect. Notes Comput. Sci. 10434, 174-181 (2017).

53. M. Kirby et al., "Total airway count on computed tomography and the risk of chronic obstructive pulmonary disease progression," Am. J. Respir. Crit. Care Med. 197(1), 56-65 (2018).

54. J. M. Bland and D. G. Altman, "Statistical methods for assessing agreement between two methods of clinical measurement," Lancet 327(8476), 307-310 (1986).

55. M. R. Spiegal and L. J. Stephens, Schaum's Outline of Theory and Problems of Statistics, McGraw-Hill Education, New York (1998).

56. D. L. Streiner, G. R. Norman, and J. Cariney, Health Measurement Scales: A Practical Guide to Their Development and Use, Oxford University Press, Oxford (2015).

57. A. de Soyza et al., "RESPIRE 1: a phase III placebo-controlled randomised trial of ciprofloxacin dry powder for inhalation in non-cystic fibrosis bronchiectasis," Eur. Respir. J. 51, 1702052 (2018).

58. J. D. Chalmers et al., "Characterization of the "frequent exacerbator phenotype' in bronchiectasis," Am. J. Respir. Crit. Care Med. 197(11), 1410-1420 (2018).

59. R. Cardenes, H. Bogunovic, and A. F. Frangi, "Fast 3D centerline computation for tubular structures by front collapsing and fast marching," in IEEE Int. Conf. Image Process. (2010).

60. D. Jin et al., "A robust and efficient curve skeletonization algorithm for tree-like objects using minimum cost paths," Pattern Recognit. Lett. 76, 32-40 (2016).

61. A. P. Kiraly et al., "Three-dimensional path planning for virtual bronchoscopy," IEEE Trans. Med. Imaging 23(9), 1365-1379 (2004).

62. Z. Xu et al., "A hybrid method for airway segmentation and automated measurement of bronchial wall thickness on CT," Med. Image Anal. 24(1), 1-17 (2015).

63. F. Grélard et al., "Precise cross-section estimation on tubular organs," Lect. Notes Comput. Sci. 9257, 277-288 (2015).

64. B. L. Odry et al., "An evaluation of automated broncho-arterial ratios for reliable assessment of bronchiectasis," Proc. SPIE 6915, 69152M (2008).

65. J. Tschirren et al., "Intrathoracic airway trees: segmentation and airway morphology analysis from low-dose CT scans," IEEE Trans. Med. Imaging 24(12), 1529-1539 (2005).

66. D. A. Moses et al., "Peripheral bronchial identification on chest CT using unsupervised machine learning," Int. J. Comput. Assisted Radiol. Surg. 13(9), 1379-1395 (2018).

67. P. Thévenaz, T. Blu, and M. Unser, "Interpolation revisited," IEEE Trans. Med. Imaging 19(7), 739-758 (2000).

68. S. Gu et al., "Computerized identification of airway wall in CT examinations using a 3D active surface evolution approach," Med. Image Anal. 17(3), 283-296 (2013).

69. A. P. Kiraly et al., "Boundary-specific cost functions for quantitative airway analysis," Lect. Notes Comput. Sci. 4791, 784-791 (2007).

70. O. I. Saba, E. A. Hoffman, and J. M. Reinhardt, "Maximizing quantitative accuracy of lung airway lumen and wall measures obtained from X-ray CT imaging," J. Appl. Physiol. 95(3), 1063-1075 (2003).

71. J.-P. Charbonnier et al., "Improving airway segmentation in computed tomography using leak detection with convolutional networks," Med. Image Anal. 36, 52-60 (2017).

72. Z. Bian et al., "Small airway segmentation in thoracic computed tomography scans: A machine learning approach," Phys. Med. Biol. 63(15), 155024 (2018).

73. Z. Yang, H. Jin, and J. H. Kim, "Attenuation profile matching: an accurate and scan parameter-robust measurement method for small airway dimensions in low-dose CT scans," Med. Phys. 45(9), 4145-4157 (2018).

74. J. Tschirren, T. Yavarna, and J. M. Reinhardt, "Airway segmentation framework for clinical environments," in The Second Int. Workshop Pulmonary Image Anal. (2009).

75. S. Žabić et al., "A low dose simulation tool for CT systems with energy integrating detectors," Med. Phys. 40(3), 031102 (2013).

76. T. Takenaga et al., "A computer simulation method for low-dose CT images by use of real high-dose images: a phantom study," Radiol. Phys. Technol. 9(1), 44-52 (2016).

77. K. C. Won and J. H. Kim, "Realistic simulation of reduced-dose CT with noise modeling and sinogram synthesis using DICOM CT images," Med. Phys. 41(1), 011901 (2014). 
78. R. E. Naziroglu et al., "Simulation of scanner- and patient-specific low-dose CT imaging from existing CT images," Phys. Med. 36, 12-23 (2017).

79. X. Li et al., "Pediatric MDCT: towards assessing the diagnostic influence of dose reduction on the detection of small lung nodules," Acad. Radiol. 16(7), 872-880 (2009).

80. M. Robins et al., "How reliable are texture measurements?" Proc. SPIE 10573, 105733W (2018).

81. P. P. Dendy and B. Heaton, Physics for Diagnostic Radiology, Taylor \& Francis, Boca Raton (1999).

82. D. G. Parr et al., "Influence of calibration on densitometric studies of emphysema progression using computed tomography," Am. J. Respir. Crit. Care Med. 170, 883-890 (2004).

83. B. C. Stoel et al., "Quality control in longitudinal studies with computed tomographic densitometry of the lungs," Proc. Am. Thorac. Soc. 5(9), 929-933 (2008)

84. J. D. Chalmers et al., "The bronchiectasis severity index an international derivation and validation study," Am. J. Respir. Crit. Care Med. 189(5), 576-585 (2014).

85. J. R. Hurst, "Microbial dysbiosis in bronchiectasis," Lancet Respir. Med. 2(12), 945-947 (2014).

Kin Quan received his MSc degree in mathematics from the University College London. $\mathrm{He}$ is a $\mathrm{PhD}$ student studying medical imaging at the University College London. His research concerns measuring and interpreting the geometry of airways in CT.

Ryutaro Tanno received his BSc and MASt degrees in pure mathematics from the Imperial College London and University of Cambridge, respectively, and his MPhil degree in computational neuroscience from the University of Cambridge. $\mathrm{He}$ is a Microsoft research $\mathrm{PhD}$ scholar. His interests are designing methods that can cheaply and reliably enhance the quality of medical image data. $\mathrm{He}$ has worked as a researcher for Mircosoft, ThinkSono, and Butterfly Network.

Rebecca J. Shipley received her first class honours master's degree in mathematics, before completing her DPhil (PhD) working on multiscale mathematical models of blood flow and drug delivery in vascularized tissues. She is a reader in biomechanics at the University College London. Her research involves computational modeling in biology and healthcare with a focus on tissue engineering and cancer. Currently, she is a director of the UCL Institute of Healthcare Engineering.

Jeremy S. Brown is an academic respiratory consultant with a subspecialty interest in lung infection. He trained in respiratory medicine in London alternating with Wellcome-funded laboratory research at Imperial College into respiratory pathogens. He has led a laboratory investigating respiratory pathogens at the University College London since 2003 and been a clinical consultant at the University College Hospitals with a special interest in patients with respiratory infection and bronchiectasis.

Joseph Jacob trained as a radiologist at Kings College Hospital with subspecialist chest radiology training at Imperial College London where he completed his MD (research). His research interest is computer-based analysis of CT imaging in lung disease for which, in 2018, he was awarded a Wellcome Trust Clinical Research Career Development Fellowship. He is based at the Center for Medical Image Computing and the Respiratory Medicine Department of the University College London.

John R. Hurst is a professor of respiratory medicine at the University College London. He has clinical and research interests in bronchiectasis and COPD. He qualified from the University of Edinburgh Medical School in 1997 and has worked at UCL since 2007. He has national and international roles with the American and British Thoracic, and European Respiratory Societies and in 2019 will become the editor in chief of the European Respiratory Monograph.

David J. Hawkes received his BA degree in natural sciences (physics) in 1974 from Oxford and his MSc degree in radiolobiology from Birmingham in 1975. He is a professor of computational imaging science at UCL. His research interests are focused on both fundamental research in medical image computing and transfer of advanced computational imaging technologies across the whole spectrum of patient management from screening to diagnosis, therapy planning, image-guided interventions, and treatment monitoring. 